\title{
DIE SECHSTAKTER \\ IN DER ALTENGLISCHEN DICHTUNG.
}

II.

\section{Allgemeiner teil.}

\section{Bisherige ansichten über den $6 \mathrm{~T}$.}

1823. J. Bosworth, The Elements of Anglo-Saxon Grammar, London.

B. will p. 247 , wie er sagt nach Turner, die längere halbzeile auf zwei gew. V zurückführen. Hiergegen wendet sich schon 1826 Conybeare in seinen Illustrations p. XII.

1826. J. J. Conybeare, Illustrations of Anglo-Saxon Poetry, London.

C. ist sich des vorkommens von versen verschiedener länge wohl bewufst. Eine genaue scheidung der verse ist indes für ihn nicht möglich, da er sie nur nach dem worttreff zerlegt. p. IX $\mathrm{f}$.: "It is to a metre ..., in which emphasis ... holds the place of quantity, that I would refer the verses of the Anglo-Saxons." p. XI: "Variety was produced, and the labour of versification lessened by the admitting lines of different lengths from two to four feet ..." p. XIII gibt C. eine gruppe der längeren verse (Genesis $B 252 b-258 a$ ) als halbzeilen von drei und vier fülsen. Anderseits hat er unter den beispielen für "shorter kind of metre" sowohl drei- als zweifülsige verse.

1830. Erasmus Rask, A Grammar of the Anglo-Saxon Tongue, translated by $B$. Thorpe, Kopenhagen.

R. bestimmt das wesen des "Long Narrative Verse" aus der stellung der stäbe. So ergibt sich seine auffassung als 
ähnlich der später von Cremer (s. u.) auf dem boden der typentheorie vertretenen. Bei R. vgl. p. 160: "We have here in the first line of each couplet, three ictus, besides a number (3-6) of short syllables, especially between the first and second ictus. In the second line are only two ictus at the conclusion, but preceded by a very long complement of from four to eight short syllables, which usually makes the second line of each couplet as long as the first: it has moreover a half ictus in the beginning ... The first line has always two sub-letters at the two first ictus. The second line has its chief letter at the first ictus; that is, in the middle of the line, after the complement, very seldom in the beginning, where the weaker emphasis, or half ictus is found." Dals die stabstellung für R.'s ansicht mafsgebend gewesen, geht noch aus seinen folgenden einwendungen gegen Conybeare hervor (p. 161). - Vgl. z.t. das unten Cremer gegenüber bemerkte.

1838. Edwin Guest, A History of English Rhythms, 2 bde., London.

Foster (vgl. besond. teil, Judith) erklärt p. 33 von den "Expanded Lines": "Among English scholars Guest has called them 'Cædmon's longer rhythms'." Diese behauptung ist insofern ungenau, als Guests "longer rhythms" sich nicht mit den "expanded lines" decken können. Genauer auf G.'s unterscheidung der verse einzugehen eruibrigt sich, da er sie willkürlich nur nach dem worttreff (vgl. oben Conybeare; diese einteilungen nach dem worttreff allein erinnern trotz ihrer hier noch sehr rohen form schon an die verslehre von Sievers, vgl. daher auch das zu dieser unten bemerkte) zerteilt, indem er einfach nach [!] jedem stärkeren worttreff, also meist mitten im wort, einen einschnitt macht. So gelangt er überhaupt nicht zu festen grenzen. Im ersten band p. 163 sagt $G$. von der halbzeile ("section"): "For the most part these sections contain two or three accents, but some are found containing four or even five." Die letzteren sollen nun eine verdopplung der ersteren darstellen, weil sie nach G. "generally fulfil all the conditions of an alliterative couplet [= langzeile]". - Im zweiten band p. 38-45 trägt jede seite am kopf die worte "Cædmon's Longer Rhythms", während es vorher und nachher "Cædmon's Rhythms" heilst. Dort finden sich nun die verse 
356-438 a der Genesis (B). Die verse in dieser stelle gehören aber in wirklichkeit nur $\mathrm{zu}$ einem kleinen teil der längeren versart an: nur aus einem ihm gewordenen allgemeinen eindruck heraus, dafs hier mehr als an anderen stellen längere verse hervorträten, hat $G$. gerade diese stelle "longer $r$." getauft; p. $36 \mathrm{f}$. sagt er vor ihrer wiedergabe: "We will ... pass ... to the speech of Satan. Here Cædmon lengthens his rhythms, and assumes greater pomp of language."

1839. J. A. Schmeller, Über den Versbau in der alliterierenden Poesie, besonders der Altsachsen; Abhandl. der philosoph.-philol. Klasse der Kön. Bayerischen Akademie der Wissenschaften 1847, gelesen am 4. Mai 1839.

Schmeller sagt hinsichtlich der "vier tonhebungen", die nach ihm die langzeile hat: "Es finden sich deren zuweilen weniger, oft mehr als vier, so dals die wahl gelassen ist, welche man als die notwendigen, welche als die müfsigen betrachten wolle." Dafs und wieviel hebungen mehr als im gewöhnlichen falle ein vers habe, ist also nach Sch. zufall. Grundsätzlich nimmt er für alle verse gleiche hebungenzahl an, und wahrscheinlich begreift seine definition der langzeile bei ihrer geringen bestimmtheit auch die $6 \mathrm{~T}$ ein und hält sie für wesentlich den gew. $\mathrm{V}$ gleichwertig. Demgegenüber vgl. das unten an verschiedenen stellen bemerkte.

1868. Heyne, Beowulf, 2. Auflage. Ist mir nicht zugänglich gewesen, daher folgendes nach schriftlichem auszug von herrn geheimrat prof. Trautmann.

Heyne erkennt unsere verse bereits als besondere art und gibt ihnen auch sechs hebungen.

1870. Jessen, Grundzüge der altgermanischen Metrik, ZfdPh II 114-147.

Jessen hängt der vierhebungen- als viertaktlehre an. Über die längeren verse spricht er sich nur unbestimmt aus. Im Althochdeutschen finden sie sich nicht, wohl aber im Altsächsischen und Altenglischen. Über ihren bau sagt er p. 138: "Die längere versart möchte vielleicht eigentlich dieselbe sein [wie die kürzere], nur mit den drei (zwei) stäben auf vier statt auf zwei glieder verteilt, und so dafs das vierte glied aus- 
fallen kann? Vgl. gesett hæfde he hīe | swā gesæliclīce \| ænne hæfde hē | swā swī̄ne geworhtne, was viergliedrig sein möchte, wohingegen stormas $\succsim æ r$ stānclýfu bēotan \| ठær him stearn oncwæठ als um ein glied abgekürzt aufzufassen sein möchte."

1870. Schubert, De Anglo-Saxonum arte metrica, Berlin; von Kaluza besprochen.

§ 9: "Exstant in Anglosaxonicis carminibus praeter ternaria et quaternaria hemistichia longiora quoque, quae, quum omnibus legibus, quas superioribus paragraphis exposui, respectis sex ictus habere possint, pars autem non plus sex, pars non minus, pro senariis habenda sunt." Es folgt liste der $6 \mathrm{~T}$ aus Beowulf, Genesis und Elene. $\mathrm{Zu}$ beachten sind noch folgende bemerkungen: "Neque modo acervatim, verum etiam passim et singulatim eius generis versus occurrunt ... Atque quum in singulis, quos adhuc attuli, versibus longis utrumque hemistichium senarium sit, non desiderantur versus longi, quorum alterum hemistichium quaternarium, alterum senarium sit." Tr in ABbl V 94 verweist bereits auf Schuberts ansicht als eine richtige; dgl. später in BB XVII 188.

1872. Vetter, Zum Muspilli und zur germanischen Allitera. tionspoesie, Wien; von Kaluza besprochen.

Vetter gibt p. 38 dem "durch einen zusatzstab vermehrten" verse drei hebungen im gegensatz zum gew. V, der für ihn zwei hebungen hat. Einen gew. $V$ trennt er vom anfange des längeren verses aus ab. Die einwände gegen diese art der zerlegung mache ich unten bei der besprechung von Luicks ansicht, da sie Luicks eingehenderer behandlung gegenüber fruchtbarer gestaltet werden können. - Hier möchte ich nur etwas von Kaluza nicht besprochenes berühren. Vetter sieht im längeren vers eine weiterentwicklung und regelung des "überfüllten", d.i. des besonders in den aufser-altenglischen denkmälern sich findenden gew. $V$ mit unregelmäIsig vielen senkungsilben. Diese auffassung geht grundsätzlich weiter als die später von Sievers geäufserte ansicht, dafs gew. V und schwellverse nicht immer streng zu scheiden seien. In der tat aber entspringen beide meinungen der unsicherheit der zweihebungentheorie. Aufserdem konnte Vetter zu seiner ansicht leicht kommen, da er zunächst die besonders unregelmälsigen 
althochdeutschen verse behandelt, wo sich dem beobachter die wahren verhältnisse verschleiern können. Trotzdem ist unbegreiflich, dals $\mathrm{V}$. den längeren vers aus dem unregelmälsigen gew. $V$ einfach weiterentwickelt wissen will, da er doch erkennt, dafs es sich im ersteren nicht um "regellose füllung" handelt: das zeigt ihm die stabverteilung und das sinngemälse beisammenstehen dieser verse. Vor allem mufste ihm bei einer betrachtung aller denkmäler der umstand zu denken geben, dals in den denkmälern mit "überfüllten" gew. $V$ auch die "dreistabigen verse" überfüllt erscheinen, wenn man sie mit denen der metrisch guten denkmäler vergleicht. Von Vetters standpunkt aus wäre diese merkwürdige entsprechung nicht denkbar, denn sie lälst deutlich für die gesamtheit der hier in betracht kommenden altgermanischen dichtung zwei wesentlich verschiedene versarten erkennen.

1873. Francis A. March, A comparative Grammar of the Anglo-Saxon Language, New York.

Part IV "Prosody" p. 222-228. Im wesentlichen richtige auffassung des gew. V ("The common narrative verse has four feet in each section", p. 225) wie des längeren verses. Über diesen, den "Long Narrative Verse", sagt March p. 227: "The common narrative verse is varied by occasional passages in longer verses. The alliteration and general structure of the long verse is the same [stabung?] as of the common; but the length of the section is six feet. Feet are oftenest added between the two alliterating syllables of the first section, and before the alliterating syllable of the second section." Der letzte satz, der uns Marchs bemerkung p. 228 "This verse is rather a variety of the Common Narrative than another kind" erklärlich macht, ist allerdings anzufechten, denn der $6 \mathrm{~T}$ ist eine selbständige versart (vgl. unten ansicht des vf.).

1876. Rieger, Die alt- und angelsächsische Verskunst, ZfdPh VII.

p. 56 ff. spricht Rieger "von der senkung". Es gibt für ihn nur zweihebige verse. Längere verse, die nicht mit einem stab beginnen, läIst er einen - allerdings geschwellten auftakt haben; solche, die mit einem stabe beginnen, haben nach Rieger eine "geschwellte" senkung. Einen vom gew. V wesentlich verschiedenen vers gibt es für ihn nicht; dagegen 
sagt er wohl, dafs eine "richtige auswahl" der durch die anschwellung sich ergebenden "mannigfaltigsten kombinationen" "sache des kunstgefühles ist, auf deren glücklicher abwechselung die äufsere formschönheit der dichtung beruht" (p. 62). Riegers zerlegung jedes beliebigen verses in zwei fülse nur nach der stellung der stäbe ist eine rohere form der annahme einer gleichwertigkeit von gew. $V$ und längerem, wie sie später Kauffmann (s. u.) auf dem boden der typentheorie gemacht hat.

187\%. Ten Brink, Geschichte der englischen Literatur I 59-60.

In ten Brinks ansicht ist unbegründet 1. dafs er im gebrauch der längeren verse eine freiheit erblickt, die durch ihre natur nicht ursprünglich sei, 2. was er über die grundlage behauptet, auf der von dieser freiheit gebrauch gemacht werden könne. "In der behandlung des epischen verses scheint die geistliche dichtung schon frühzeitig eine freiheit sich gestattet $\mathrm{zu}$ haben, die sogar in die überlieferung der jüngeren teile des volksepos, wenigstens in die interpolationen der redaktoren eingang fand. Der streng rhythmische vortrag der epischen sänger gestattete ihnen zwar in der ausdehnung des verses, d. h. in der zahl der versfüfse hinter dem metrischen schema zurückzubleiben, wo dann durch längeres verweilen auf gewissen silben oder durch pausen das zeitmals ausgefüllt wurde, nicht jedoch es zu überschreiten. In den geistlichen epen aber, die wahrscheinlich einfach rezitiert, nicht gesungen wurden, konnte die tradition, welche das zuwenig erlaubte, leicht dahin führen, auch das zuviel sich zu gestatten. Daher denn hier die verse manchmal über das mafs der acht hebungen hinausschwellen, zwar innerhalb bestimmter grenzen, doch eine grofse mannigfaltigkeit der formen erzeugend, die nur [!] durch die lage der drei reimstäbe unter die einheit eines gesetzes gebracht werden." Das oben gegen ten Brinks ansicht bemerkte erhellt daraus, dafs nichts für, wohl aber die einheitliche geltung der rhythmischen gesetze gegen die annahme verschiedener vortragsweise für "volks"- und geistliches epos spricht. Dann fällt aber der grund, in den längeren versen etwas nicht ursprüngliches zu sehen; gegen diese annahme spricht übrigens wohl schon innerhalb der altenglischen dichtung das vorkommen dieser verse in den Denksprüchen. Endlich und vor allem aber sind die längeren verse kein beliebiges "hinausschwellen über 
das mals der acht hebungen", kein "zuviel", sondern eine selbständige, in sich fest geschlossene versart, deren takte den takten des gew. $V$ vollkommen gleich sind, deren anwendung also dem "streng rhythmischen vortrag der epischen sänger" nicht entgegen war.

1878. A. Fritzsche, Das angelsächsische Gedicht Andreas und Cynewulf, A II.

p. 470 f. redet Fritzsche von unseren versen, die er langverse nennt. "Wir verstehen hier darunter einen vers, in welchem an einer oder mehreren stellen die äufserste zulässige zahl der senkungen angewendet wird, so dafs ein unschöner [!] vers herauskommt." Also gleichwertigkeit des längeren verses mit dem gew. $V$, wie andere sie zu beweisen versucht haben, s. u. Man wird Fritzsche zugeben, dals es nicht schön ist, wenn die höchste zahl der senkungsilben von der letzten hebung des anverses bis zur ersten des abverses zehn ist, wie er herausrechnet.

1886. Luick, Über den Versbau des angelsächsischen Gedichtes Judith, PBB XI 470-492; von Kaluza besprochen.

Nach Luick, der auf den typen von Sievers (PBB X) aufbaut, sind "die streckverse der mehrzahl nach gegen die cäsur hin erweiterte A-verse, derart dals in der ersten hälfte an das A-schema $\dot{-} \times$ oder $\times \dot{-} \times$ antritt, in der zweiten dasselbe mit mehrsilbigem auftakte versehen wird." Unrichtig ist zunächst die meinung, der anvers beginne mit einem gew. $V$ und auf diesen folge eine erweiterung. Kaluza hat diese ansicht widerlegt (gegen Vetter, p. 346). Nicht immer könne das am anfang abgetrennte stück grammatisch als ein zusammengehöriges ganzes empfunden werden, wenn $z$. b. in einer halbzeile die beiden letzten starktonigen wörter die beiden teile eines kompositums oder adjektiv + substantiv oder genetiv + regierendes substantiv sind; aufserdem sei das abgetrennte stück oft metrisch zu kurz. Dies hat schon Luick gefühlt, und so macht er seine durch die verschiedene erklärung von an- und abvers schon künstliche theorie noch geschraubter dadurch, dals er die mit abtrennung eines gew. $V$ vom anfang aus unmöglichen verse als " $\mathrm{D}$ " "-verse anders unterbringt, $z$. b. Judith 17 a bealde

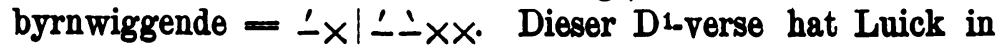


der Judith 13 gegen 51 "F"-, d. h. gegen die cäsur hin erweiterte verse. Willkürliche annahmen führen also hier in ihren folgen zu gänzlicher zersprengung des metrischen systems. - Bei der erklärung des abverses ist Luick der mehrsilbige "auftakt" gefährlich geworden. Für die verse mit zweisilbigem Vst hält er es für möglich, dafs man sie "als übergangsform ... betrachten mufs". So kommt es, dals er auch in versen mit einsilbigem auftakt, d. i. wirklichem vorschlag, eine übergangsform erblickt. Hier zeigt sich sehr deutlich, dafs mit der annahme eines "mehrsilbigen auftaktes" eine feste begrenzung der längeren verse nicht möglich ist aus dem grunde, dals eine solche bestimmung das wesen dieser verse verkennt: sie sind mehr als eine beliebige silbenansammlung plus gew. V.

Hier seien gleich Luicks spätere ansichten behandelt. 1888. Zur Theorie der Entstehung der Schwellverse, PBB XIII 388-392; von Kaluza besprochen.

Luick versucht hier eine neue erklärung, wobei er ausgeht von der ihm merkwürdigen tatsache, dafs die erste hebung im zweiten halbvers eine "sekundäre" sei, nicht aber im ersten halbvers. Es ist ihm deshalb nicht möglich, mit Sievers den anvers ebenso aus den "normaltypen" entstanden zu denken wie den abvers. L. erklärt den anvers so, dals er mit einem normaltypus beginne; "mit der zweiten hebung jedoch tritt eine abfolge ein, als ob sie die erste hebung irgend eines der fünf typen wäre". Bei der zweiten hebung des zuerst angesetzten verses sei "das erregte gefühl noch nicht befriedigt". So ist es ihm erklärlich, dals die ersten zwei hebungen des neu entstandenen gebildes staben, denn sie waren ursprünglich zwei erste hebungen. Es ergeben sich so für Luick theoretisch fünfzehn formen, von denen aber praktisch nur sechs vorkommen. "Diese erklärungsweise scheint mir den vorteil $\mathrm{zu}$ besitzen, dals mittelst einer und derselben operation alle formen sich ergeben und dafs die stellung der alliteration ihre begründung findet." Im zweiten halbverse trete nur eine sekundäre hebung vor die normaltypen. "Einem gewissen metrischen symmetriebedürfnis" habe es nämlich entsprochen, "nachdem die tonstärke im ersten halbvers langsam gesunken war, nun allmählich zur tonstärke des hauptstabes hinanzusteigen, und so entstand die sekundäre hebung". Dann aber 
sei es zu spät gewesen, erst wieder jene umbildungen vorzunehmen. Mit recht hat Kaluza auf das mechanische dieser erklärung hingewiesen und bemerkt, wenn das mit einem normaltyp noch nicht befriedigte "erregte gefühl" den dichter bei der zweiten hebung genötigt habe, von neuem anzufangen, wie Luick dies will, so könne man eine reihe von aufeinander folgenden längeren versen nicht erklären, denn dort könne doch der dichter nicht bei jedem einzelen verse mit einem gew. V haben beginnen wollen und sich erst mitten in diesem eines besseren besonnen haben. Das mifsliche an Luicks erklärung ist ihr ganz gekünstelter charakter; ihre vorteile sind weit einfacher zu gewinnen. Für die stabverteilung hat uns Luick selbst später den weg zu einer erklärung gewiesen. Keinesfalls darf der blofse umstand, dafs die stabverteilung im anvers eine andere ist als im abvers, zu einer verschiedenen erklärung beider halbzeilen veranlassen angesichts der tatsache, dafs die stäbe nicht etwas dem verse von vornherein wesentliches sind.

1891. Zur altenglischen und altsächsischen Metrik (Schwellvers und Normalvers, Alliteration und Versrhythmus), PBB XV 441-454; von Kaluza besprochen.

Hier ist $\mathrm{L}$. davon abgekommen, den abvers anders als den anvers zu erklären, d. h. er lärst ihn ebenso künstlich wie diesen durch wiederholtes ansetzen entstanden sein. "Nur überwiegt im zweiten [halbvers], was im ersten selten ist: dals die zweite hebung allein alliteriert und dem entsprechend die erste hebung minder stark ist. Die ursache dieser erscheinung scheint mir in eigentümlichkeiten der $z$ weiten halbzeile und des stabverses überhaupt zu liegen" (p. 448). "Wir bemerken eine neigung, im ersten halbvers das hauptgewicht an den anfang zu verlegen, ... und im zweiten halbvers das hauptgewicht dem schlufs zuzudrängen, obwohl die erste hebung die stärkere ist. Also eine neigung zu jener symmetrie, von der ich Beitr. XIII 391 sprach, besteht tatsächlich und dürfte jene verschiebung in den schwellversen schon begreiflicher erscheinen lassen." Das vorhandensein der "neigung" $z u$ besagter gewichtsverteilung wird durch die tatsachen sofort bewiesen. $\mathrm{Ob}$ aber die neigung sich als jenes "symmetrie- 
bedürfnis" erklärt, sei vorläufig dahingestellt. Der abvers hat also ein stabwort ziemlich in der mitte. Und nur so kann er mit dem einzigen stab auskommen. Denn es gibt "eine grenze, bis zu welcher sich der stab vom versende entfernen kann; die fälle des ersten halbverses, welche über sie hinausgehen würden, zeigen durchgehends doppelalliteration." Der grund, warum auf die stabsilbe nur noch eine beschränkte zahl stabloser silben folgen kann, "ist offenbar der, dafs der reimstab ein gröfseres mals von silben und akzenten nicht mehr übertönen kann und dadurch der vers seinen halt verliert. Dies erklärt, warum in den schwellversen der zweiten halbzeile normalerweise die zweite hebung alliteriert: anders war es nicht möglich, einen vers von drei hebungen und nur einem stabwort so zu bauen, dafs der stab die zeile bis zu ihrem ende beherrschte." (Dieser gedanke nach Luicks angabe p. 454 ohne sein wissen auch schon in einem brief von Sievers vom 11. IV. 1887 ausgesprochen). - Um Luicks ausfïhrungen zusammenfassend $\mathrm{zu}$ betrachten, sei wiederholt: hauptgewicht im anvers nach vorn, im abvers nach hinten. Das von Luick zur erklärung dieser tatsache angenommene "metrische symmetriebedürfnis" liegt m. e. nicht vor. Den kern der sache hat hier wohl Deutschbein, Zur Entwicklung des englischen Alliterationsverses, 1902, p. 7 f., getroffen: zur verbindung der langzeilen wird der sogenannte hakenstil angewant; es wird also durch "enjambement" der abvers mit dem folgenden anvers syntaktisch verbunden, während in sich die langzeile durch den stabreim gebunden ist. So ergibt sich dann durch die satzkonstruktion das "steigen" des den gedankenfortschritt bringenden abverses und das "fallen" des die weiterführung bezw. den abschlufs des gedankens bringenden anverses. In Luicks ausführungen ist dann wieder, wenigstens im kerne, richtig, dals bei diesem charakter des anverses seine zunächst natürliche stabsilbe am anfang nicht genügt, während der abvers, der seinem charakter gemäls keine stabsilbe am anfang hat, wenigstens eher von seinem weiter nach hinten stehenden stab ganz übertönt werden kann. Dies gesetz von der "übertönung" ist indes Luick gegenüber noch genauer festzulegen, was unten in der darlegung meiner ansicht geschehen soll. 
1887. Sievers, Zur Rhythmik des germanischen Alliterationsverses, III. Der angelsächsische Schwellvers, PBB XII 454-482; von Kaluza besprochen.

Dem gew. V gibt Sievers zwei hebungen; dem "schwellvers" gibt er deren drei. Seine auffassung dieses verses hat vor der anderer forscher den vorzug, dals sie ihn nicht zwingt, die einheitlichkeit des altgermanischen rhythmus durch den schwellvers durchbrochen sein zu lassen. Diese grundforderung erfüllt S. Im einzelen aber krankt seine auffassung der längeren verse am mangel an bestimmtheit. Hierfür mögen seine eigenen worte sprechen (p. $457 \mathrm{f}$.): "Eine vollkommen sichere scheidung der schwellverse von den normalversen ist einstweilen nicht möglich, ehe wir nicht über das mafs der in den einzelnen dichtungen vorkommenden lizenzen der normalverse genau unterrichtet sind. Die grenzen zwischen den (z. b. durch auftakt) zu möglichster länge erweiterten normalversen und den denkbar kürzesten schwellversen werden sich bis dahin nicht abstecken lassen; fraglich ist es mir sogar, ob dies überhaupt einmal möglich sein wird. ... Pafst ein schwerer gang des verses am besten für eine in frage stehende stelle, so wird schwellvers anzunehmen sein, andernfalls ein blos zweifülsiger vers mit stärkerem (zweisilbigem) auftakt, gröfserer silbenzahl in den inneren senkungen u. dgl. Dals bei solchen entscheidungen das individuelle rhythmische gefühl des einzelnen stark mitspricht, bedarf keines beweises. Meine aufstellungen in diesem punkte machen daher keinen anspruch darauf, für absolut angesehen zu werden." S. wird also durch seine metrik vielfach über den charakter der verse im zweifel gelassen, und nur "das individuelle rhythmische gefühl des einzelnen" soll dann entscheiden können. Aber die wahre verskunst bietet dem individuum zu solcher entscheidung keine gelegenheit, weil es "stärkeren auftakt, gröIsere silbenzahl in den inneren senkungen u. dgl." nicht gibt. Zweifellos ist mit der anwendung der längeren verse ein bestimmter stilistischer zweck verbunden. Dieser kann aber nur erreicht werden, wenn metrisch jeder längere vers streng von dem gew. V gesondert ist. Herrscht die scheidung nicht für alle verse, so herrscht sie überhaupt nicht, und jenes "individuelle rhythmische gefühl" könnte dann schliefslich alle verse als gew. V nehmen. Auch in metrisch schlechten gedichten waren 
ursprünglich gew. $V$ und längere verse wohl geschieden, und auch heute noch mufs in ihnen aus dem gesamtbilde der gew. $V$ einerseits und der längeren verse anderseits über die natur des einzelen, wenn auch schlechten verses entschieden werden können. Da ein dichter sicher bei keinem seiner verse die entscheidung über dessen hebungzahl jemand freistellen wollte, sondern in jedem verse eine ganz bestimmte zahl von hebungen beabsichtigte, so wird diejenige verslehre vor der von Sievers den vorzug verdienen, die von ihren eigenen grundsätzen aus die beiden versarten zu scheiden vermag. In Pauls Grundrifs II 875 weisen S.'s eigene worte auf den besprochenen mangel seines systems hin: "Im einzelnen sind sie [die "schwellverse"] nicht immer mit voller sicherheit von den normalversen $\mathrm{zu}$ unterscheiden, da ihre kürzesten formen mit den längsten formen der normalverse äufserlich, wenn auch nicht ihrem wahren rhythmus nach, zusammenfallen." Warum aber dann nicht die verse "ihrem wahren rhythmus nach" bestimmen? - Eine verwischung der grenzen ist es auch, wenn S. in seiner Altgerm. Metrik $\S 96$ neben den dreihebigen schwellversen auch noch seltene vierhebige teils fest, teils für möglich annimmt. Alle diese verse sind $6 \mathrm{~T}$, allerdings einige, besonders in den Denksprüchen, von ungewöhnlichem, meist verderbtem bau.

Bei ihrer geringen festigkeit vermag die verslehre von Sievers nicht nur nicht gew. $V$ und längere verse streng zu scheiden, sondern sie weils auch nicht die für sie sicheren schwellverse stets metrisch richtig wiederzugeben. Dies zeigt S.'s liste: in textkritischer hinsicht mufste diese unzureichend sein durch die schuld der für die textkritik nicht genügend fruchtbaren, weil zu dehnbaren theorie. Wie die bearbeitung der verse im einzelen zeigt, sind S.'s vers-"füfse", besonders der erste, vielfach zu lang.

Da bei S.'s wenigen hebungen die stabstellung sich zu sehr hervordrängt und den vers bestimmt, so schwankt S. in einigen fällen aufsergewöhnlicher stabstellung, ob er den ersten stab zum Hst schlagen oder das hierdurch dann meist leichte Vst einfach als "auftakt" eines "normalverses" nehmen soll (p. 467, absatz zwei).

Zur feststellung der grenze zwischen V'st und Hst - dies als einem "normalvers" gleich genommen - ist die verslehre 
von Sievers unfähig, wo das Hst für S. ein typus B oder C ist. Beim schwellvers mit typus $\mathrm{C}$ hat $\mathrm{S}$. unmögliche Vst und Hst dadurch, dals alle auf die erste hebung folgenden für $\mathrm{S}$. hebunglosen silben zur "inneren senkung", d. h. nach dem p. 469 gegebenen schema zum Hst gezogen werden: letzteres wird so zu lang. Beim Hst = typus B sucht S. eine mögliche einteilung $\mathrm{zu}$ gewinnen, indem er die senkungsilbe(n) des ersten fufses des B-verses zum Vst schlägt, wodurch aber wieder unmögliche abteilungen, nämlich zu kurze Hst, sich ergeben. - Ja, wenn bei diesen typen nicht ein stab den "schwellvers" beginnt, so ist offenbar überhaupt keine grenze mehr zwischen geschwelltem und einfachem typus $C$ bezw. B, was S. p. 470 wenigstens für $C$ zugibt; allerdings sucht er auch hier nach scheidenden merkmalen, doch ist hier auf dem boden seiner verslehre eine reinliche scheidung gar nicht möglich, weil doch nach dieser verslehre im gew. $V$ wie im längeren. schliefslich das, was in den typen $C$ und $B$ der vorletzten hebung voraufgeht, ein "silbenhaufen", nicht aber eine für beide versarten verschiedene, durch eine takteinteilung in festen grenzen gehaltene silbenzahl ist.

188\%. Frucht, Metrisches und Sprachliches zu Cynewulfs Elene, Juliana und Crist; Diss. Greifswald.

Im allgemeinen ist Frucht Luicks älterer ansicht. Der eingang hat eine hebung im anvers, im abvers ist er teils mit teils ohne hebung. Wo aber so die halbzeile dreihebig ist, da ist immer eine, meist die letzte hebung, eine nebenhebung. Eine bemerkung erfordert nur folgendes: "Ich verstehe unter streckversen zunächst verse, welche in beiden hemistichen eine ungewöhnliche erweiterung zeigen. Dieselben erscheinen sowohl gruppenweise als auch einzeln. Aufserdem bezeichne ich als streckverse auch diejenigen verse, welche zwar nur in einem hemistich ungewöhnlich erweitert sind, aber zwischen streckversen der erstgenannten art stehen. Dagegen scheinen mir auf den namen streckverse keinen anspruch zu haben verse, welche sich unter gewöhnlichen versen befinden und nur in ihrem einen hemistich eine solche ungewöhnliche erweiterung aufweisen." Dieser "schein" hält vor der verslehre nicht stand, denn sie weist auch vereinzelte halbzeilen als unzweifelhafte $6 \mathrm{~T}$ aus. 
1888. Cremer, Metrische und sprachliche Untersuchung der altenglischen Gedichte Andreas, Guthlak, Phönix, (Elene, Juliana, Crist); ein Beitrag zur Cynewulffrage, Diss. Bonn; von Kaluza besprochen.

Die auffassung Cremers hat mit der von Luick gemein, daIs die verteilung der stäbe für sie von grundlegender bedeutung ist. Blofs hat Luick darüber hinaus zu einer einheitlichen auffassung aller längeren verse zu kommen gesucht, während davon bei C. nicht die rede sein kann. C. stimmt mit Sievers "darin überein, dals viele schwellverse (besonders in der ersten halbzeile) dreifüfsig sind und zwar die, welche die stäbe im ersten und zweiten fufse haben. Alle anderen halte ich für normale $\mathrm{A}, \mathrm{D}$ oder $\mathrm{E}$ typen, die durch mehrsilbigen auftakt geschwellt sind. Diese art der schwellverse ist für die zweite halbzeile regel (ausnahmen sind sehr selten), begegnet aber seltener im ersten halbverse, der meistens dreifülsig ist" (p. 25). Ausschlaggebend ist also für Cremers doppelte erklärung die stellung der stäbe: nach ihm wollte der dichter alle dem stab vorausgehenden silben "als unbetonte, als auftaktsilben aufgefaIst wissen". Dem widerspricht aber schon der umstand, dafs ein längerer vers mit einem zwar nicht stabenden, aber doch als vorschlag viel $\mathrm{zu}$ schweren worte beginnen kann, das sogar an sich des stabes fähig wäre. Derartige vorschläge sind hierdurch und zugleich durch ihre länge jedem rhythmischen gefühl zuwider. Bei verschiedener stabstellung sind die längeren verse rhythmisch alle gleich lang: sie werden eben nicht durch die für den vers als solchen ganz unwesentliche stabstellung, sondern durch die takteinteilung mit sicherheit bestimmt.

1889. H. Hirt, Untersuchungen zur westgermanischen Verskunst, Heft 1: Kritik der neueren Theorieen, Metrik des Angelsächsischen; Leipzig; von Kaluza besprochen.

Nach Hirt hat der gewöhnliche anvers drei oder vier, der abvers drei hebungen. Ähnlich schreibt er im "schwellverse" dem anvers fünf, dem abvers vier oder fünf hebungen zu. Der grund dieses völligen mangels an einheitlichkeit liegt darin, dals Hirt sich bei der metrischen bestimmung der verse zu sehr von den wortformen beeinflussen lälst; so ist in seinen versen nur der schlufs einigermalsen bestimmt. 
1891. Kauffmann, Die sogenannten Schwellverse der altund angelsächsischen Dichtung, PBB XV 360—376; von Kaluzu besprochen.

p. 361: "Mit versformen dieser art [typen D und E] teilen die sogenannten schwellverse merkmale allernächster verwandtschaft." Für die schwellverse scheine "die lizenz zu gelten, dafs sowohl im zweiten als auch im ersten halbvers ein vollwertiges begriffswort ohne reim [folglich ohne volle hebung im verse?] den übrigen stabwörtern vorausgeht". p. 364: "Das charakteristische schema des dreigliedrigen fufses der D-verse, dessen grundform von Sievers als $\dot{-} \dot{*} \times$ oder $\dot{-} \times \dot{-}$ dargestellt worden ist, hat umbildungen $z u \stackrel{\prime}{\prime} \times x^{\prime}$ und $\dot{\prime} \times \dot{-} \times$ erfahren." Vor allem zeige der ausgang der geschwellten halbzeilen den charakter gesteigerter D-verse; p. 365: "Der versschlufs der halbzeilen in den sog. schwellversen ist von dem der normalen D-verse nicht verschieden." Seine vorgänger hätten bereits die von ihnen angenommene dritte hebung nur als nebenhebung betrachtet, er ziehe die schlufsfolgerung, "dafs diese dritte vershebung überhaupt nicht existiere." p. 366: "Mit andern worten: die sogenannten schwellverse sind nichts anderes als gesteigerte Dverse." Weiter p. 371, dals "alle verse, in denen die zweite 'und dritte' hebung ohne die erste alliterieren, nicht als sog. schwellverse zu betrachten sind, sondern als A-verse mit auftakt." p. 372: "Im zweiten halbvers scheinen die ausnahmen des ersten [dals nicht die erste hebung und vor allem dafs nur eine stabe] die regel zu bilden." Regelrecht müsse auch im abverse die erste hebung den stab tragen. Deshalb seien die nach ansicht von Sievers regelmälsigen zweiten halbzeilen mit stab auf $z$ weiter hebung gerade die unregelmäIsigen, sie zeigten "entartung der technik". p. 373: "Ich halte es für wahrscheinlicher, dals die betr. verse als A mit auftakt resp. B rezitiert worden sind." - Gegen Kauffmann wandte sich Luick in seinem besprochenen dritten aufsatz. Er wies nach: "Die versschlüsse der schwellverse sind nicht identisch mit den schlüssen der D-verse ... Der brauch der dichter zeigt uns deutlich, dafs und wo eine grenzlinie zu ziehen ist. Nur $\dot{\prime}^{-}-x, \stackrel{\prime}{-} \times \dot{\prime}$ und $\dot{\prime} \times x \dot{\prime}$ sind schlüsse von $\mathrm{D}$, alle anderen sind ausschliefslich den schwellversen eigen" (p. 444). Ferner wendet sich Luick gegen die von Kauffmann 
behauptete zweihebigkeit. Der typus D sei scharf abgegrenzt, weil die zweite hebung nur ein gewisses ausmals von senkungsilben und nebentönen tragen könne. Wenn die hebung nicht mehr imstande sei, über den nebenton zu dominieren, dann hätten wir "eine dritte hebung vor uns, wenn sie auch an tonstärke den ersten beiden nachsteht." Endlich wendet sich Luick gegen Kauffmanns auffassung des zweiten halbverses, der keine andere entstehung habe wie der erste. - Kaluzas kritik (p. 340) schliefst sich wesentlich an Luick an. - Es ist unnötig, die unmöglichkeit einer ansicht, die sich vom standpunkt der wenig bestimmten zweihebungen-theorie aus dartun lälst, noch besonders zu beweisen.

1892. Foster, Judith studies in Metre, Language and Style, Diss. Stralsburg; von Kaluza besprochen.

Wie Foster p. 33 dartut, steht er in seiner auffassung vom wesen der "Expanded Lines" auf dem boden der verslehre von Sievers. - Unzulässig ist es, dafs Foster eine ihm zufolge namentlich in den späteren gedichten vorkommende längere form von den übrigen expanded lines besonders unterscheidet als "longer type", denn solche verse, die sich nicht der strengen verskunst entsprechend lesen lassen, sind fast stets schlecht überliefert, in einigen wenigen fällen vielleicht metrisch schlechte erzeugnisse einer entarteten kunst und können daher keinen anspruch erheben, als besondere gattung aufgefalst zu werden. - Übrigens können Fosters auf dem grunde von Sievers aufgebaute metrische anschauungen nicht das wesentliche der übernormal langen verse erfassen. Nach Foster besteht nämlich ihre "expansion" aus "a chiefstressed and three or more secondary- or unstressed syllables". Es können demnach auch in wirklichkeit regelmäIsige Vst für Foster dem longer type angehören, während seine bestimmung die mit zu langem oder zu schwerem Vst natürlich in eine reihe zu setzenden zu langen oder zu schweren Hst nicht einschliefst. Der ganze unterschied zwischen gewöhnlichen und längeren expanded lines ist so, wie $F$. ihn behauptet, überhaupt nicht vorhanden. F. scheint zu seiner annahme einer früheren kürzeren und einer späteren längeren expanded line wie auch zu der anderen annahme von der seltenheit der 
exp. lines in den "certainly oldest poems" gekommen $\mathrm{zu}$ sein durch die vorhergehende, von ihm nach ten Brink vertretene annahme, dals in der ältesten dichtung exp. lines aus technischen gründen der vortragsart unmöglich gewesen seien; sie träten also erst allmählich auf und hätten sich dann später weiter in die länge entwickelt. Über die grundlosigkeit dieser annahme vgl. das oben ten Brink gegenüber bemerkte.

1892. K. Fuhr, Die Metrik des westgermanischen Alliterationsverses, Marburg; von Kaluza besprochen.

Über den "schwellvers" p. 87: "Im Angelsächsischen begegnen uns viele verse, die sich in ihrem baue von normalen nur dadurch unterscheiden, dafs der nach der füllung des zweiten taktes zu erwartende stumpfe ausgang durch klingenden ersetzt wird ... Die "schwellung" solcher verse besteht demnach in der vermehrten schwere des zweiten taktes." Gegen Fuhrs auffassung wendet sich Kaluza p. 343. Fuhr - der der vierhebungen-theorie anhängt - verteile die hebungen genau wie Koegel (s. u.). Er gebe nicht an, "aus welchem grunde und unter welchen bedingungen" jener ersatz eintrete. "Überdies ergeben sich bei skandierung der schwellverse nach Fuhr genau dieselben übelstände, die ich oben bei Koegel hervorgehoben habe [s. u.]; es ist also auch Fuhrs erklärung der schwellverse unzulänglich, zumal sie überhaupt nur für einen teil derselben anwendbar ist."

1893. H. Frank Heath, The Old English Alliterative Line; Transactions of the Philological Society 1891/93, p. $375 \mathrm{ff}$.; Sitzung vom 2. Juni 1893. Bisher mir nicht zugänglich gewesen, daher folgendes nach Kaluza p. $351 \mathrm{f}$.

Heath ist für die "Expanded Lines" im wesentlichen Luicks erster ansicht: erweiterung in an- und abvers gegen die pause hin; doch gibt er dem gew. $V$ vier, dem längeren sechs hebungen. - Ähnlich wie Sievers neben den dreihebigen auch einige vierhebige schwellverse annimmt, so gibt es für Heath aufser versen, die "expanded by a half" (p. 386) sind, auch verdoppelte gew. V: "This type is the double of the normal 
line" (p. 391); sie findet er besonders in der Genesis B. Diese weicht aber auch in metrischer hinsicht von der übrigen altenglischen dichtung ab, kann also zur aufstellung einer solchen allgemeinen regel nicht herangezogen werden; im übrigen aber ist zu Heaths annahme kein anlals.

1894. Heusler, Über germanischen Versbau, Berlin; von Kaluza besprochen.

Kaluza hat zwar p. $344 \mathrm{f}$. die unmöglichen folgen von Heuslers ansicht dargetan, dafs sich nämlich auch bei ihm undenkbare versschlüsse ergeben, ist aber nicht auf Heuslers verfehlten beweis für die gleichwertigkeit von gew. V und längerem vers eingegangen. Kurz ist Heuslers gedankengang dieser (p. $101 \mathrm{ff}$.): die grundmeinung von Sievers: "Weil der sprachkomplex zweigipflig ist, ist es auch der vers; wäre der sprachkomplex dreigipflig, so müfste es auch der vers sein" ist irrig. Es mufs vielmehr heifsen: "Der vers, seinem metrischen rahmen nach, ist zweigipflig; infolgedessen wird der sprachkomplex zweigipflig rhythmisiert." Heusler führt dann gew. $\mathrm{V}$ an, die sprachlich dreigipflig, rhythmisch zweigipflig seien, z. b. scencte scīr wered könne rein sprachlich sehr wohl dreigipflig gemessen werden; also sei hier zu einem zweitaktigen verse ein dreiteiliger sprachkomplex verwendet worden. Man wird Heuslers satze (p. 103): "Nicht zweigipflige silbengruppen brauchte der dichter, sondern solche, die sich zweigipflig rhythmisieren liefsen" vom standpunkte der zweihebungen-theorie aus, allerdings nicht in Heuslers sinne, beipflichten, indem man auf der grundlage von Sievers hinzusetzt: "Nicht jede silbengruppe aber lälst sich zweigipflig rhythmisieren, sondern nur eine solche, die an ausdehnung gewisse grenzen nicht überschreitet." Und auf grund dieses zusatzes wird man Heuslers behauptung p. $104 \mathrm{f}$. verwerfen: "Können die schwellverse ebenso wie ihre ungeschwellten nachbarn zweitaktig rhythmisiert werden? - diese frage kann auf grund unserer kenntnis des alten satztones mit sicherheit bejaht werden." Wenn nun auch, wie schon angedeutet, Sievers sich gegen eine solche ausdehnung der zweihebigheit auf die "schwellverse" auf grund der von ihm über die füllung der senkung aufgestellten sätze sperren würde, so ist doch nicht $\mathrm{zu}$ verkennen, dafs $\mathrm{H}$. hinsichtlich dieser füllung nur 
einen schritt weiter geht als Sievers. Dieser schon zergliedert unter weitgehender mifsachtung der silbenzahl und -schwere den vers nach wort- und satztreff. H. will dann nur noch vom satztreff etwas wissen und teilt jeden vers nach zwei satztreffen. Die letzte folgerung wäre, dafs man erklärte: "Von diesen zwei satztreffen ist einer der stärkere; man kann also den anderen ihm gegenüber vernachlässigen und so auf grund des satztones jeden vers einhebig rhythmisieren." Was aber wäre dann nicht vers? - Man wäre damit am ende alles rhythmus angelangt und würde dann vielleicht in die engen grenzen zurückkehren, die durch die bedingungen des wahren rhythmus dargestellt werden: worttreff und silbendauer sind die wahren grundlagen des altenglischen versbaus. - Die quelle aller schwierigkeiten ist auch bei Heusler die abneigung, für die altgermanische dichtung verse verschiedener taktzahl anzunehmen. Diese wären zwar für $\mathrm{H}$. nicht wie für Kauffmann ein zeugnis niederen kunstsinnes, sie sollen aber dem altgermanischen metrischen stile nicht entsprechen. p. $107 \mathrm{f}$.: "Dals schon die stabreimenden Germanen dieses ausdrucksmittel [der freien taktzahl] gepflegt hätten, entspricht wenig dem stile ihrer verstechnik." Einen beweis bringt $H$. nicht: "In der versgeschichte wie anderswo führt weitere historische betrachtung zu gewissen unterscheidungen, gewissen wahrscheinlichkeitssätzen, die man im einzelnen falle nicht immer beweisen kann, deren leitung auf unsicherm boden man doch nicht verschmähen wird" (p. 108). Wenn sich nun auch Heusler gegen die bewertung seiner ansicht durch Sievers ("lediglich axiomatische gründe") verwahrt, so ist doch zu sagen, dals er auf höchst unsicherer, a priori angenommener grundlage aufbaut, die vollends vernichtet wird durch das zutagetreten der auf ihr sich ergebenden unmöglichen versgestalten.

1894. Koegel, Geschichte der deutschen Literatur bis zum Ausgange des Mittelalters; von Kaluza besprochen.

p. $288 \mathrm{n}$ betrachtet Koegel die von Sievers " als notwendige senkungen bezeichneten glieder seiner schemata als schwächere hebungen", also den gew. $V$ als vierhebig. "Rhythmisiert man den sog. 'normalvers' vierhebig, so fällt natürlich jeder grund weg, ihn von dem gleichfalls vierhebigen 'schwellverse' zu 
scheiden. Beide sind bis auf die verschieden starke taktfüllung durchaus identisch." Zunächst vermag Koegel mit seiner erklärung nicht alle 'schwellverse' unterzubringen (p. 306). Im übrigen ist $z \mathfrak{u}$ bemerken: von den vier hebungen läIst $K$. den zwei am ende eines 'schwellverses' stehenden silben zwei (vgl. p. 289). So entfallen die beiden ersten hebungen auf eine derart grofse anzahl von silben, dafs der vers sich in zwei höchst ungleiche hälften scheidet. "Verschieden starke taktfüllung" kann es nun aber beim wesen des taktes nur in höchst beschränktem umfange geben. Die störung des rhythmus wird aber um so fühlbarer dadurch, dals unter jenen beiden am ende des verses je eine hebung tragenden silben keine stabsilbe ist, also zwei takte auf nur zwei und dazu verhältnismäIsig leichte silben entfallen, während die beiden übrigen takte von mindestens doppelt so vielen und schwereren silben ausgefüllt werden und so die gewichtigen stabsilben in den durch Koegels lesung um die beiden ersten hebungen sich ergebenden zusammenballungen von silben ganz erdrückt werden. - Diese einwendungen gegen Koegels ansicht ähnlich auch von Kaluza.

1894. Trautmann, Zur Kenntnis des altgermanischen Verses, vornehmlich des altenglischen, $\mathrm{ABbl} \mathrm{V} \mathrm{87-96;} \mathrm{von}$ Kaluza besprochen.

Trautmann erklärt die sogenannten streck- oder schwellverse für sechstakter, als welche sie schon Schubert (s. 0.) richtig erkannt habe. Dasselbe tut er 1905: Die neueste Beowulfausgabe und die altenglische Verslehre, BB XVII 175191. Hier p. 188 der zusatz: "Jeder sechstakter besteht aus irgend einem [halb-]verse unsrer $16+12$ gestalten und einem zweitaktigen vorstück, das vorschlag haben oder auch nicht haben kann."

Andere äufserungen Trautmanns über den sechstakter liegen gedruckt nicht vor; ausführlicher behandelt er den vers in seinen vorlesungen über altenglischen versbau (vgl. einleitung $\mathrm{zu}$ dieser arbeit).

Kaluzas einwendungen gegen Trautmann erledigen sich durch meine behandlung von K.'s auffassung. 
1894. Franck, Beiträge zur Rhythmik des Alliterationsverses, ZfdA XXXVIII 225 ff.; von Kaluza besprochen.

Franck hält es p. 238 für unwahrscheinlich, das man den "schwellvers" als einen "fremden vers eingemischt" habe. "Also mülste der schwellvers denselben grundvers in einer anderen entwicklung, die sich aus den vorliegenden tatsachen begreifen liefse, darstellen. Und das tut er in der tat; er ist, wie es gar nicht anders zu erwarten ist, derselbe vers in einer anderen vortragsweise." Die schwellverse hätten etwas feierliches durch ihre getragene vortragsweise, in ihnen hätte die alliteration nicht dieselbe wucht wie im gewöhnlichen verse, und darum könnten alle hebungen eher ihren vollen wert behalten. Nun legt auch Franek zwei von den vier hebungen des verses auf seine zwei letzten silben und gibt zu, dafs dieser rhythmus nicht immer "in der wortbetonung eine stütze finden wird, dann nämlich nicht, wenn das letzte wort einen logisch deutlich untergeordneten ton hat. Der rhythmus wird dann wohl die neigung haben, sich anders zu verteilen, nämlich die beiden ersten wörter als die rhythmisch höchsten werte einander entgegenzusetzen. Dann ergibt sich zwischen den beiden eine pause, das dritte wort ordnet sich im tone dem zweiten unter, und seine letzte silbe wird überschüssig". "Ich verhehle mir nicht die schwierigkeit, welche darin liegt, daIs hier hinter $\stackrel{-}{ } \times$ eine verwendung von $\dot{-} \times \mathbf{z u -}$ gelassen wird, die wir beim gewöhnlichen alliterationsvers nur hinter 1 finden. Weiter muls zugestanden werden, das sekundär das erste glied stärker gefüllt werden konnte als wir es für den ursprünglichen vers annehmen." "Jene beiden betonungstypen mülste man nebeneinander annehmen." Kaluza (p. 344) bringt gegen F. das beweismittel vor, dafs "am versschlufs nach langer stammsilbe eine senkungssilbe in der gesamten altgermanischen metrik unzulässig ist". Der von Franck daneben angenommene "betonungstyp" mit stärkerer füllung des ersten gliedes ist auch unmöglich. F. ist zu dieser annahme genötigt, um den silbenstoff der längeren verse unterzubringen. Dieser versuch mufs indes daran scheitern, dals im vergleich zum gew. $V$ der silbenstoff des längeren verses zu grofs und zu schwer ist für die gleiche zahl von hebungen (vgl. Koegel). Der grundirrtum Francks, dessen notwendige 
folgerungen diese rhythmisch unhaltbaren anschauungen sind, ist die von ihm behauptete unwahrscheinlichkeit der einmischung "fremder" verse.

Als charakteristisch für Francks auffassung sei hier noch folgende stelle (p. 241) wiedergegeben: "Wir hätten also im schwellverse gewissermaisen den rest einer älteren stufe der entwicklung. Die alliteration hat sich dem gewöhnlichen verse gesellt, ihn auch schon etwas beeinflufst, aber nicht so stark .wie später. Es scheint mir eine natürliche entwicklung, wenn diesem stadium ein anderes folgte, in dem der zufällige schmuck ein mehr selbständiges leben gewann und stark umgestaltend auf das organ wirkte, dem er sich gesellt hatte. In dem neuen stadium erhielt der vers etwas leidenschaftliches und gewaltsames; für gewisse stimmungen bewahrte sich noch die ältere, getragenere art daneben. Aber das gefühl für die gleichartigkeit beider war noch nicht abgestorben, man konnte aus der einen art zwanglos in die andere übergehen ... So kann ich mir die erscheinung der schwellverse erklären; als eine versart von ganz anderem ursprung vermag ich das nicht." Der stabreim kann, eben weil er ein "zufälliger schmuck" des verses war und blieb, nicht so stark auf den ursprünglichen vers, der nach Franck der später seltene längere vers gewesen sein soll, eingewirkt haben, dafs sich ein rhythmisch mit ihm gar nicht unter einen hut $\mathrm{zu}$ bringendes gebilde ergeben hätte.

1895. Kaluza, Die Schwellverse in der altenglischen Dichtung, ESt XXI 337-384.

Kaluza vertritt die vierhebungen-theorie in einer ihm eigentümlichen form (kritik von $\mathrm{Tr}$ in $\mathrm{ABbl} V$ 131-136). Bei seiner erklärung der "schwellverse" lälst Kaluza sich von zwei gesichtspunkten leiten (p. 354): alle schwellverse seien einheitlich zu erklären und bei strenger scheidung von gew. V und schwellvers dürfe "der übergang von der einen versart zur anderen nicht erschwert" werden. Bei seinem versuch, alle längeren verse in gleicher weise zu erklären, geht er aus von der mehrheit der abverse, in denen der vers nicht mit dem stab beginnt. Hier will er nun dem "eingange" keinen takt zusprechen, weil es "bei der wechselnden länge Anglia, N. b. XXI. 
des einganges der schwellverse unmöglich ist, demselben eine bestimmte zahl von hebungen zuzuweisen" (p. 378). Nach Kaluza kann allerdings der eingang eine bis fünf und noch mehr silben haben; in wirklichkeit aber ist sein "einsilb. eingang" einfacher vorschlag oder, wo K. vor ihm einen "auftakt" annimmt, zweisilbig, und die mehr als viersilbigen eingänge lassen sich kürzen, ja fordern vielfach wie so mancher gew. $V$ zur kürzung geradezu heraus (vgl. das bearbeitete material). Es gibt also nur eingänge, um hier. diesen unguten ausdruck K.'s noch zu gebrauchen, von zwei bis vier silben. Sollen die nun nicht alle eine gleiche zahl von takten füllen können? Sicher können sie dies, können doch auf den gew. $V$, der stets gleich viele takte hat, vier bezw. drei bis acht silben entfallen. Also schon für die stablos anfangenden verse, wo K.'s erklärung noch am wenigsten unberechtigt erscheint, ist nicht zu beweisen, dals der eingang taktlos sein müsse. Die stabend anfangenden verse, also die minderheit der ab- und die mehrheit der anverse erklärt dann K. (p. 379 f.) ebenso wie die eben besprochenen: "Wir müssen eben dabei berücksichtigen, dals der vers das ältere, die alliteration das jüngere ist, ein äufserer schmuck des verses ..." Dies ist richtig, doch erhalten den schmuck nur solche wörter, die an sich schon ein gewisses gewicht - metrisch = taktfüllung - haben, das durch den stab noch deutlicher hervortritt. Und nun sollte ein nomen, das in jedem gew. $V$ ganz abgesehen vom stab eine hebung tragen mü Iste, im "eingange" hebunglos sein! Dies ist nicht einzusehen, um so weniger als die unmöglichkeit gleichzahlhebiger "eingänge" und damit der hebungen im eingange überhaupt gefallen ist. So ist es ein schlufs aus falscher voraussetzung, wenn K. p. 381 behauptet: "Weil dort der erste reimstab auf ein im auftakt [der nach $K$. hebunglos sein mufs] stehendes wort fällt, ist er schwächer als gewöhnlich ..." Falsch ist es, dafs der eingang "blofser erweiterter auftakt" sein soll, und nur dieser annahme zuliebe mufs für $\mathrm{K}$. jedes wort im eingange des treffes entkleidet werden.

Aber auch die stablosen eingänge - und hier stolsen wir auf den m. e. mit der rhythmik unvereinbaren kern in K.'s schwellvers-erklärung - können der hebungen, der einteilung in takte gar nicht entbehren. So gut 
wie im gew. $V$ gar mancher takt von ungewichtigen silben und wörtchen, da diese doch einmal so gut vorhanden sind wie die gewichtigen, gefüllt werden mufs, mufs dies auch in jenen "eingängen" der fall sein. Die hauptforderung aller verskunst ist doch die, dafs aller sprachliche stoff, der überhaupt mit rhythmischen werten verbunden werden soll, aus dem stets gleichen, einheitlichen rhythmischen gefühl heraus behandelt werden mufs. Wenn nun im gew. V stets hebungen sich nach ganz bestimmten gesetzen in ganz bestimmten intervallen wiederholen, so sorgt das rhythmische gefühl dafür, dafs diese wiederholung überall in gleicher weise sich vollziehe. Dies gefühl kann die ihm entsprechende wellen- oder schaukelbewegung von hebung $\mathrm{zu}$ senkung und wieder $\mathrm{zu}$ hebung $\sim$ nicht auf einmal aufgeben und gewissermalsen in der ebene fortschreiten, und umgekehrt kann es nicht aus gleichmäIsigem fortschreiten in wellenbewegung übergehen, falls nicht die voraussetzung der fortdauer seiner ihm natürlichen wellenbewegung aufhört. Diese voraussetzung ist aber stets gegeben durch das vorhandensein von wörtern, die einer hebung überhaupt nur fähig sind.

Mit dieser letzten erörterung werde ich auch K.'s forderung gerecht, der übergang von einer versart zur anderen dürfe nicht erschwert werden. K. hält mit Kauffmann die "unsymmetrische" einmischung von versen mit einer der gewöhnlichen nicht gleichen hebungzahl für ein "zeugnis niederen kunstsinnes". Nach dem eben gesagten wird man indes in solchen versen keine "störenden stromschnellen" (Kauffmann) erblicken. Diese vorwürfe sind vielmehr gegen verse zu erheben, in denen die sonst allgemein durchgeführte rhythmische wellenbewegung jäh unterbrochen würde. Der dichter will durch die form bestimmte wirkungen erzielen; gut: er darf dazu ein mittel wählen, das nicht den rhythmus vernichtet. Ein solches mittel ist die anwendung von versen mit $a+b$ statt der gewöhnlichen a takte. Nicht "planlos" (K. p. 349) wechseln innerhalb desselben gedichtes verse verschiedener taktzahl, sondern das ist eben das "planvolle", dals ein vers, der sich vor einem anderen hervorheben soll, mehr takte erhält als dieser. Nach allem gesagten wirklich planlos und zugleich eine vernichtung der poetischen kunstform wäre es dagegen, wenn der dichter rhythmisch gehobene stücke (in "schwellversen" abtrennbare 
gew. V) mit beliebig langen stückchen prosa ("eingänge") wechseln liefse.

1895. Schipper, Grundrifs der englischen Metrik, Wien und Leipzig.

Über den "schwellvers" § 28-30. Ein auszug aus den darlegungen von Sievers. Hinsichtlich des "baus, resp. der entstehung des schwellverses" entscheidet Schipper nicht zwischen der auffassung von Sievers und Luicks annahme der vers-verschmelzung, schliefst sich aber dieser, wie auch Sievers tut, "mit rücksicht auf die allerdings zweckmäLsige schematische bezeichnungsweise" an.

\section{0\%. Martin, Der Versbau des Heliand, Strafsburg.}

Martin nimmt gleichwertigkeit von gew. $V$ und "schwellvers" an. Die schwellverse sind für ihn (p. 3) "rhythmisch nicht unterschieden von den normalversen". Für M. besteht die langzeile aus (p. 59) "je zwei halbversen zu vier versgliedern, von denen je zwei in freiem wechsel über die zwei andern durch stärkere betonung erhoben werden". p. 60: "Steht der hauptstab auf dem 1 . versglied, so kann dieses bis auf 5 silben ausgedehnt werden, das 2 . bis auf 3, das 3 . ebenso, aber nur ganz selten. Der vor dem 1 . versglied stehende auftakt kann bis auf 11 [!] silben steigen. Die erste vershälfte hat seltener und nur mäfsigeren, bis auf 5 silben steigenden auftakt; das 1. versglied kann dafür bis zu 9 silben anschwellen, das 2 . weit seltener bis auf 4, das 3 . hat höchstens 2 silben, 3 nur in zweifelhaften fällen. Alle diese angeschwellten verse dienen fast stets zum ausdruck gehobener, feierlicher stimmung, besonders bei lehren." p. 65: "Diesem rallentando des schlusses steht gegenüber das accelerando des verseingangs und das ad libitum des auftaktes, der vor dem 2. halbvers einen so gewaltigen umfang annehmen kann." Es ist nämlich für M. (p. 65) "eine wenigstens annähernd gleiche dauer der versglieder wahrscheinlich". p. 68: "Völlig gleiche dauer solcher bald aufgeschwellter, bald auf das knappste mafs beschränkter verse kann natürlich nicht behauptet werden; nur dafs der viergliedrige vers auch in seinen freien gestaltungen doch als solcher noch gefühlt wurde." Einen beweis für die behauptete gleichwertigkeit aller verse bringt 
M. nicht; wir haben aber gesehen, wie vieles dagegen spricht. Bei der dehnbarkeit von M.'s verslehre - sein "auftakt" kann viel länger werden als der ganze vers, vgl. Tr's kritik in BB XXIII $148 \mathrm{f}$. - lä Ist sich verstehen, dafs er die längeren verse von den gewöhnlichen nicht $\mathrm{zu}$ scheiden vermag.

190\%. Saran, Deutsche Verslehre $=$ Handbuch des deutschen Unterrichts III 3, München.

Daraus hier einschlägig $\S 27$ (die Alliterations-Metra) p. 222-235; $§ 28$ (zur Geschichte der altgermanischen Verskunst) p. $235-243$.

p. 226: "Normalvers' und 'schwellvers' haben sich entwickelt jeder aus einem geschlossenen, strengen, einfachen, taktmäfsigen urrhythmus ..." Der urrhythmus macht verschiedene wandlungen durch. Der überlieferte 'normalvers' hat fïr Saran zwei haupthebungen, der 'schwellvers' drei; zu lesen sind die zeilen "wie pathetische, schwungvolle, rhythmische prosa". Diese versart soll (p. 225) "ihren eigenartigen rhythmus einer art literarischer revolution verdanken." Über den schwellvers p. 232 ff. Indem er betont, hier nur schematisch zu verfahren, teilt S. den schwellvers in einen vom schlufs aus abgetrennten und nach den typen von Sievers benannten 'normalvers' und ein davor stehendes stück ${ }^{-} \times$

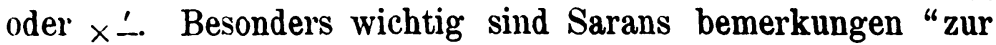
Geschichte der altgermanischen Verskunst". p. 236: "Die oben gegebenen ableitungen .. der schwellverse aus den normalversen sind logisch. Historisch sind sie nicht ..." Deshalb wird (p. 241) Luicks theorie der zusammenschiebung verworfen. "Der westgermanische schwellvers geht auf den urrhythmus von sechs hebungen zurück:

... Das längere hauptbund ist also an umfang vollkommen dem urrhythmus des normalverses gleich und macht auch in der entwicklung des metrums dieselben verwandlungen und änderungen durch wie der letztere. Das vorderbund wird grundsätzlich ebenso behandelt, wie es oben für den normalen vierer angegeben ist. Aber da eben nur ein zweier vorliegt, ferner die enge verbindung von zweier + vierer zur reihe modifikationen nahelegte, ist das ergebnis ein anderes, weniger mannigfaltiges und weniger durchsichtiges." p. 242 f.: "VIan 
vergesse aber auch hier nicht, dals ich nicht die verstexte, sondern nur die versformen historisch ableite und keineswegs annehme, die uns überlieferten schwellzeilen wären je als sechser skandiert oder gar gefühlt worden. Ihrem rhythmischen werte nach sind sie dreier bezw. vierer (letztere mit einer nebenhebung)."

Nach allem gesagten ist zu Sarans ansicht wenig zu bemerken. Für mich ist besonders wichtig, dals er entschieden die getrennte, für beide formen selbständige entwicklung von 'normal-' und 'schwellvers' (wozu dann aber noch diese namen?) betont 1) und dafs er wenigstens den urversen vier und sechs hebungen gibt. In der metrischen grundanschauung von den überlieferten formen der stabdichtung mufs ich von Saran abweichen: in ihnen lägen uns nach ihm aus den urrbythmen durch eine literarische revolution entwickelte zwei- und dreihebige verse vor (ähnlich Sievers, Altgermanische Metrik abschnitt VII). So verfällt Saran der unbestimmtheit in der auffassung der verse, der schwierigkeit, die versarten zu scheiden, wie sie oben Sievers gegenüber gekennzeichnet worden ist. Hier mufs ich also im gegensatz zu Saran betonen, dals ich im überlieferten versmaterial sämtliche urtakte noch verwirklicht sehe. Denn wo ist der beweis, dafs das ursprüngliche metrische gefühl unter dem eindringen sprachlicher neuerungen in die formeln und texte unsicher geworden sei und ein neues formensystem sich aus der zerrüttung herausgelöst habe (so Saran p. 226)? Ehe dieser beweis erbracht ist, ist es mir weit wahrscheinlicher, dafs das rhythmische bewulstsein von einer bestimmten dem verse zukommenden taktzahl sich über die sprachlichen vorgänge hinaus gerettet hat.

\section{Ansicht des verfassers. Ergebnisse. ${ }^{2}$ )}

Die verse, mit denen die arbeit sich beschäftigt, sind bisher von den meisten beurteilern streck- oder schwellverse genannt worden. Schon diese namen weisen darauf hin, dafs man die verse nicht als ein metrisch selbständiges und unabhängiges

1) Meine eigene hervorhebung der selbständigkeit auch der längeren versart war bereits schriftlich niedergelegt, als ich auf Sarans werk stiefs.

2) Der $6 \mathrm{~T}$ eine selbständige versart. - Bau des $6 \mathrm{~T}$. - Die stäbe im $6 \mathrm{~T}$. - Anwendung der $6 \mathrm{~T}$. $-6 \mathrm{~T}$ und Verfasserfragen. 
gebilde behandelte, sie vielmehr von einem anderen herleitete. Und zwar legte man ihnen den sogenannten normalvers zugrunde. Unter diesem verstand man die bei weitem hänfigere kürzere form des stabverses, über deren bau man im übrigen keineswegs einer meinung war. Besser als mit dem ausdruck "normalvers", der wieder den längeren vers als etwas unselbständiges, ja unregelmäLsiges erscheinen lassen könnte, wird man die zahlenmäIsig häufigere kürzere art des stabverses als "gewöhnlichen vers" ansprechen. Die erklärungweisen, die den "schwellvers" als einen durch vor- oder ansetzung eines stïckes erweiterten gew. $V$ darstellen, sind rein mechanisch und wollen z. t. auch nichts anderes sein. Zu solcher erklärung verführte der umstand, dals der längere vers wie der kürzere dem ja einheitlichen rhythmus unterliegt. Es war also mechanisch möglich, von dem längeren verse einen gew. $V$ irgendwie abzuschneiden. Aber aus dem gew. $V$ entstanden ist der längere nicht. Dals der längere vers als selbständiges gebilde neben dem gew. $V$ steht, wird durch verschiedene umstände bewiesen. Auch im besonderen teil dieser arbeit ist in den längeren versen hinten ein stück als gew. $V$ abgetrennt, doch soll damit nur eine mechanische zerlegung aus sozusagen technischen gründen gegeben sein. Bereits oben (Luick gegenüber) ist dargelegt worden, dafs diese abtrennung am ende auch die einzig mögliche mechanische zerlegung liefern kann, dals eine abtrennung vom anfang des längeren verses aus nicht möglich ist. Unsere mechanische zerlegung zeigt uns nun, dafs das vom längeren vers als gew. $V$ abgetrennte stück zwar eine form des gew. $V$ in jedem einzelen falle, nicht aber auf die gesamtheit dessen durchschnittsform ist. Warum sollten aber bei vorsetzung eines stückes vor den gew. $V$ nicht dessen formen in ihrer gewöhnlichen relativen häufigkeit verwendet werden? Die durchschnittsform des abtrennbaren gew. $V$ kommt mehr als die des freien gew. $V$ der hinsichtlich der silbenzahl und -schwere kürzesten form nahe, die der gew. $V$ haben kann, d. h. die verstreffe folgen einander in verhältnismälsig kurzen abständen. Dieser letzte ausdruck weist auf eine strengere und eine weniger strenge taktfüllung hin, und diesen unterschied gibt es, wenn auch in sehr engen grenzen: es ist vom gew. $V$ bekannt, dafs die festigkeit seines baues gegen den schlufs hin zunimmt. Der im längeren verse 
abtrennbare gew. $\mathrm{V}$ zeigt nun eine grölsere gedrungenheit als der freie gew. V: diese tritt uns vorzüglich darin entgegen, dafs für den anfang des abtrennbaren gew. $V$ nicht die freiheiten gelten, die dem anfang des freien gew. $V$ gestattet sind. Der erste takt des abtrennbaren stïckes kann nicht $=1 \smile$ sein, wohl aber der erste takt des freien gew. $V$ (falls dieser takt nicht den einzigen stab der halbzeile trägt). Dem abtrennbaren stïck kommt also keine selbständige geltung zu; durch die festigkeit seines baues entspricht es vielmehr etwa der mitte und dem schlufs des freien gew. V. Dies wird zur vollen gewilsheit, wenn wir das durch die zerlegung sich ergebende kleinere stück des längeren verses ansehen: es zeigt sich dem anfang des freien gew. V ähnlich durch seine im vergleich zum übrigen verse freie gestaltung; ihm kommen die freiheiten des versanfanges, der erste takt = - $\smile$ und der vorschlag zu. So ist der bau des längeren verses in seiner gesamtheit dem des gew. V entsprechend, nicht aber der bau des abtrennbaren stückes, und so weist sich der längere vers als ein aus sich selbst frei und selbständig entwickeltes gebilde aus. - Angesichts der parallelen entwicklung von gew. $V$ und längerem vers lag es für diejenigen, die dem ersteren uraltindogermanischen ursprung zuweisen, nahe, für den letzteren ein gleiches $\mathrm{zu}$ tun; das tut, allerdings nicht mit bestimmtheit, Sievers in Pauls Grundrifs II $875 \S 24$. Ein so hohes alter mufs man indes wohl für beide versarten dahingestellt sein lassen; hinsichtlich des gew. V vgl. Tr in ABbl V 135.

Die nächste frage ist die nach dem bau der längeren verse im einzelen. Gehen wir vom gew. $V$ aus. Hier stelie ich ganz auf dem boden von Tr's ansicht, vgl. dessen aufsätze in $\mathrm{ABbl} \mathrm{V}$ und in BB XVII. In letzterem p. $181 \mathrm{f}$.: "Die ae. langzeile besteht aus zwei viertaktigen halbversen, von denen wir den ersten den anvers, den zweiten den abvers nennen. - Die acht takte sind jeder zweiweilig; die erste weile ist immer die hebung, die zweite die senkung. Die beiden weilen des taktes werden entweder durch zwei silben oder durch nur eine vertreten. Im ersten falle vertritt die erste die hebung, die zweite die senkung; im zweiten falle vertritt die eine silbe hebung und senkung. - Ist ein takt zweisilbig, so 
müssen beide silben kürzen sein; ist er einsilbig, so mufs die silbe eine länge sein. Nur der erste takt des halbverses darf

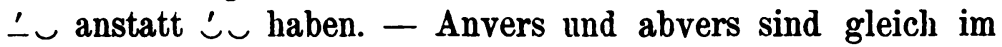
bau; doch besteht der unterschied dals der erste takt des anverses sehr häufig, der erste takt des abverses sehr selten das mals $\_$statt $\_$hat; was mit der tatsache zusammenhängt, dafs der abvers nur einen stab hat, der anvers zweie hat oder haben kann. - Das schema des halbverses ist nach dem gesagten $\dot{x} \cup \mathcal{L}^{\prime} \sim$. In diesem schema sind 16 möglichkeiten enthalten ..." p. 184: " $\mathrm{Zu}$ achten dieser 16 gestalten gibt es nebenformen, im ganzen zwelfe, denen allen eigen ist, dafs eine silbe zwei aufeinander folgende takte füllen mufs." - Für die längeren verse gilt nun zunächst, dafs sie demselben durch worttreff und silbendauer (BB XVII 186 f.) bedingten rhythmus unterliegen wie der gew. V: der rhythmus ist einheitlich. Hebung und senkung müssen also in unseren versen nach denselben gesetzen wie im gew. $V$ wechseln. Dies grundgesetz führt zur bestimmung der taktzahl des längeren verses. Greifen wir z. b. aus dem Beowulf einen beliebigen längeren vers heraus und lesen ihn nach demselben rhythmus wie einen gew. $\mathrm{V}$ :

1706, mægen mid mōdes snyttı'um. Ic pē sceal mīne gelæ̌stan. Da jeder vers gegen den schlufs hin am festesten metrisch bestimmt ist, so verteilen wir die takte über die zeile von hinten aus: -læ̈stan mufs am schlufs zwei takte füllen; mine ge- mufs zwei weitere takte füllen, $=\stackrel{-}{ }$ ‘; weiter ist dann ic pë sceal zwei takten gleich. Nach denselben taktfüllunggesetzen entfallen auf mōdes snyttrum vier und auf mægen mid zwei takte. Diese zeile hat also insgesamt zwölf takte. Ebenso wie die gewöhnliche langzeile teilt sie sich in zwei halbzeilen, deren jede hier sechs takte hat. Diese taktzahl kommt notwendig jeder anderen längeren halbzeile zu, soweit sie nicht verderbt und vor der takteinteilung durch meist naheliegende änderungen $\mathrm{zu}$ bessern ist. Das schema

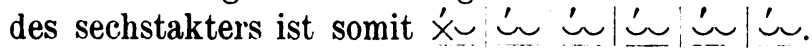

Um die zahl der möglichen formen des $6 \mathrm{~T} z u$ ermitteln, teilen wir ihn schon hier in zwei + vier takte. Für diese vier takte gibt es bekanntlich 16 möglichkeiten; die vorausgehenden zwei takte haben deren 4: das schema des 
$6 \mathrm{~T}$ enthält ihrer also zunächst 64 . Wie aber im $4 \mathrm{~T}$ so können auch im $6 \mathrm{~T}$ unter umständen zwei aufeinander folgende takte von einer einzigen silbe gefüllt werden. Hier bestehen nun für die vier letzten takte 12 möglichkeiten, von denen jede mit jeder der genannten 4 möglichkeiten der beiden ersten takte eine gestalt ergibt; so erhalten wir hier 48 gestalten. Weiter können aber die beiden ersten takte des $6 \mathrm{~T}$ von einer einzigen silbe gefüllt sein, was durch verbindung mit den 16 möglichkeiten der vier letzten takte 16 weitere gestalten ergibt. Endlich könnte eine einzige silbe den zweiten und dritten takt des $6 \mathrm{~T}$ füllen, woraus 16 weitere möglichkeiten entsprängen; dafs indes von diesen praktisch keine vorkommt, ist vielleicht nicht zufall ${ }^{1}$ ), sondern mag dem umstande entspringen, dals das beim dichter infolge der einheitlichkeit des rhythmus in den beiden versarten vorhandene gefühl für die möglichkeit der abtrennung eines $4 \mathrm{~T}$ im $6 \mathrm{~T}$ immer reger geworden war und schliefslich in einigen kleineren punkten - ein weiterer wird bei der stabstellung besprochen - zu einer andeutung der möglichen gliederung des $6 \mathrm{~T}$ führte. Jedenfalls aber sind theoretisch der von Tr "dehnverse" genannten formen für den $6 \mathrm{~T}$ insgesamt 80 möglich. Die gesamtzahl aller möglichen gestalten des $6 \mathrm{~T}$ ist somit 144.

Nun kommen schon von den gestalten des gew. V einige sehr selten vor. Von denen des $6 \mathrm{~T}$ fehlen - von jenen 16 bereits erwähnten abgesehen - viele gänzlich. Dies ist naturgemäls, da im verhältnis zu dem reichtum des $6 \mathrm{~T}$ an möglichen formen die vorhandene zahl der verse dieser art als klein bezeichnet werden mufs. Praktisch bleibt also die zahl der gestalten hinter der berechneten zahl weit zurück. Trotzdem hätte ich bei der behandlung der einzelen verse, wenn ich deren formen als ganze hätte metrisch bezeichnen wollen, mindestens $144-16=128$ gestalten und demgemäls nummern ansetzen müssen, wodurch sich ermüdend hohe ziffern ergeben hätten. Dieses praktische bedenken veranlafste mich, die zwei ersten und die vier letzten takte für sich zu bezeichnen;

1) Kann indes wohl zufall sein: füllung der beiden ersten takte des $6 \mathrm{~T}$ durch eine einzige silbe $\mathrm{z}$. b. findet sich auch nur in einem einzigen falle: Reimlied $55 \mathrm{~b}$. 
für die letzteren konnte ich Tr's numerierung des gew. $V$ verwenden. Die schematische zerteilung des verses empfahl sich auch deshalb, weil durch sie die rhythmische verwantschaft des $6 \mathrm{~T}$ mit dem gew. $\mathrm{V}$ hervortrat, weil der dichter selbst wahrscheinlich die möglichkeit der abtrennung eines $4 \mathrm{~T}$ kannte und betonte, endlich weil so die betrachtung der listen von Sievers und Kaluza vielfach anschaulicher wurde, denn in diesen ist der "schwellvers" äufserlich ähnlich wie hier abgeteilt. Die für die sich ergebenden teilstücke des $6 \mathrm{~T}$ gebrauchten bezeichnungen vorstiick und hauptstück sind $\operatorname{Tr}$ zu verdanken. An das rein schematische der zerteilung erinnere der, wo tunlich, immer wieder gebrauchte gesamtname "sechstakter".

Bei der folgenden betrachtung über das vorkommen der einzelen formen sondern wir ebenfalls Vst und Hst. So erhalten wir eine anschauung sowohl vom anfang als auch von mitte und schlufs des $6 \mathrm{~T}$. Ein bild des gesamtverses wird sich daraus ergeben.

Von den gestalten des Vst sind $1 \mathrm{~b}$ und $3 \mathrm{~b}$ vorzugsweise im anverse vertreten: ihm fallen im Beowulf 4 von 5 fällen dieser gestalten $\mathrm{zu}$, im Daniel 8 von 10. Der grund dieser verteilung liegt darin, dafs der anvers meist mit einem stabwort - d. h. einem wort von solcher schwere, dals seine sprachlich lange stammsilbe auch metrisch lang sein muls beginnt, wodurch der erste takt häufig von der form $\_$ist, während im Vst des abverses meist nicht stabtragende, vielfach leichte wörter stehen, die die schwere taktform ‘ $\smile$ nicht ergeben können. Der grund, dals $\_$den anvers häufiger als den abvers einleitet, ist also im $6 \mathrm{~T}$ ein anderer als im gew. $\mathrm{V}$ : in diesem beginnt der abvers oft mit dem hauptstabe, und lange einweilige hauptstabsilbe ist nicht zulässig. - Da von den leichten silben des abvers-Vst mehr zur füllung zweier takte gehören als von den schweren silben des meist stabtragenden anvers-Vst, so wird ferner die silbenärmere form 4 im anvers, die silbenreichere form 1 aber im abvers häufiger sein. In der tat finden wir form 1 im Beowulf fünfmal im abvers, einmal im anvers, im Andreas viermal im abvers, einmal im anvers; dagegen findet sich form 4 im Daniel zwanzigmal im anvers, siebenmal im abvers, in der Judith fünfundzwanzigmal im anvers, neunmal im abvers. Beachtens- 
wert ist, dafs sich form 4 in den Denksprüchen in abverse verhältnismälsig häufig findet (dreizehnmal gegen zwanzigmal im anvers), denn dort fällt der hauptstab, d. h. eine schwere silbe, in verhältnismälsig vielen (unter diesen 13 in 7) fällen ins Vst anstatt ins Hst, was sonst nur ausnahmweise vorkommt. Zwei solcher leichten silben, wie sie häufig im Vst des abverses stehen, reichen eben kaum aus, um am anfang des verses zwei takte zu füllen; vielfach lassen sie sich, wo sie dies scheinbar doch tun, auf drei silben ergänzen; doch darf man angesichts der tatsache z. b., dals im Daniel in sämtlichen bis auf einen von 7 und in der Genesis $A$ in sämtlichen bis auf einen von 10 fällen der form 4 als abvers-Vst leichte silben stehen, die form 4 aus solchen silben nicht ohne weiteres für falsch ansehen. - Die seltenste der formen des Vst ist form 2: wir treffen sie unter den 66 längeren versen der Genesis A in 7, unter den 135 der Judith in 12 fällen. Der hauptgrund ihres seltenen vorkommens ist der rhythmus. Wie im gew. $\mathrm{V}$ so stehen auch im $6 \mathrm{~T}$ die zweisilbigen takte am liebsten am anfang des verses; der rhythmus wird gegen den schlufs hin am festesten. Dementsprechend können wohl die beiden ersten takte des $6 \mathrm{~T} \mathrm{zu}$ gleich zweisilbig sein, nicht so gern aber läfst man auf ein $\therefore$ ein $\mathcal{L}^{\prime}$ folgen. Durch die wortform geboten ist form 2, wenn auf ein auch leichtes wort von einer silbe, das nicht vorschlag sein mufs, ein zweisilbiges folgt; ebenso ziehe ich in beiden halbversen form 2 vor, wenn von drei einsilbigen wörtern das erste schwer ist oder gar stabt und die beiden folgenden leicht sind. Gehört aber die (lange) stabsilbe einem zweisilbigen wort an, dem ein wenn auch leichtes einsilbiges wort folgt, so setze ich wegen der gröfseren rhythmischen dehnbarkeit des versanfanges form $3 \mathrm{~b}$ (lange hauptstabsilbe erfordert natürlich auch hier form 2). Zweifel, ob form 2 oder 3 anzunehmen ist, kann eintreten, wenn das Vst aus drei mehr oder minder leichten wörtchen von je einer silbe besteht, deren keines stabt: in diesem falle habe ich jedesmal versucht, die einzelen wörtchen nach ihrer doch stets etwas verschiedenen schwere möglichst genau gegeneinander abzuwägen und danach 2 oder 3 anzusetzen; doch ist wohl auf grund des genannten rhythmischen gesetzes manchmal auch unter nichtbeachtung geringer schwereunterschiede für form $3 \mathrm{zu}$ ent- 
scheiden. Zweifel, wie ein versanfang aufzufassen ist, können im $6 \mathrm{~T}$ so gut wie im gew. $\mathrm{V}$ auftreten (für diesen vgl. Tr in ABbl V 93). - Wenig ist über form $3 \mathrm{zu}$ sagen. Sie ist von allen formen die uncharakteristischte, deshalb auch neben 1 die häufigste. Ihrem auftreten steht nicht der rhythmus entgegen, nur in einem seltenen falle verbietet sich ihre annahme durchaus: wenn das Vst des abverses mit der hauptstabsilbe beginnt und diese lang ist. - Allgemein ist zum Vst noch zu bemerken, dals es syntaktisch am meisten geeignet war zur einschiebung metrisch unzulässiger und sachlich überflüssiger kleiner wörtchen, eines pronominalen subjekts, eines possessivpronomens, eines pær u. dgl. Nach deren streichung wird man nicht sagen können, dals ein denkmal mehr zur anwendung der volleren formen des Vst hinneigte als ein anderes. Nur in den $6 \mathrm{~T}$ des Traumgesichts war infolge der gedrungenen darstellung form 1 schon ursprünglich besonders häufig: zwei drittel aller Vst zeigen sie hier. - Beim Vst ist schliefslich noch der vorschlag $\mathrm{zu}$ besprechen. Er findet sich natürlich beim $6 \mathrm{~T}$ so gut wie beim gew. V. Während er jedoch bei diesem im anvers im durchschnitt etwas häufiger ist als im abvers (vgl. Tr in ABbl V 93), der häufig mit dem hauptstab beginnt und deshalb keinen vorschlag tragen darf, finden wir ihn beim $6 \mathrm{~T}$ im abverse so gut wie im anverse, weil der hauptstab nur ausnahmweise im Vst steht. Bemerkenswert ist die häufigkeit des vorschlages im Traumgesicht, wo ihn über zwei fünftel der Vst zeigen; dabei ist er im anvers überall, im abvers meistens vorsilbe eines zeitworts: die bereits erwähnte ursache ist die, dafs die art der darstellung bei der erzählung der eigentlichen handlung, die in diesem gedicht in $6 \mathrm{~T}$ gehalten wird, eine sehr gedrungene ist, in der sich die zeitwörter häufen.

Wenden wir uns den gestalten der Hst zu, so sehen wir zunächst, worauf bereits hingewiesen wurde, dals nicht diejenigen gestalten am häufigsten vorkommen, die auch als freie $4 \mathrm{~T}$ am meisten hervortreten, dafs vielmehr die durchschnittliche form des Hst der silbenärmsten möglichen form des $4 \mathrm{~T}$ näher steht als die durchschnittliche form des freien gew. V. Über die häufigkeit der gestalten des letzteren sagt $\mathrm{Tr}$ in BB XVII u. a.: "Die verschiedenen gestalten des ae. verses sind von ungleicher häufigkeit. Am geläufigsten sind 
die, in welchen zwei und drei takte einsilbig sind. Weniger häufig sind die mit vier einsilbigen takten." Gerade die gestalt mit vier einsilbigen takten ist nun im $6 \mathrm{~T}$ am reichsten vertreten. Sie umfaIst mehr als die hälfte aller Hst, in einigen denkmälern bedeutend mehr als die hälfte, so in der Judith und in "Crist III", am meisten in der Genesis A: drei viertel aller Hst. Der grund, aus dem gestalt 16 im $6 \mathrm{~T}$ so häufig ist, liegt hauptsächlich in dem bereits genannten gesetze von der festigkeit des verses gegen den versschlufs hin: im $6 \mathrm{~T}$ ist das Hst nur mitte und schlufs des gesamtverses; zweisilbige takte aber, die ja leicht $=\stackrel{-}{ }$ sein und dann überhaupt nur im verseingang stehen können, sind im $6 \mathrm{~T}$ wie im gew. $\mathrm{V}$ gewissermal'sen gegen den versanfang hin gedrängt. Nächst gestalt 16 begegnen im $6 \mathrm{~T}$ am häufigsten die gestalten mit drei einsilbigen takten, und zwar gilt hier im allgemeinen, da्s sie um so häufiger sind je näher der zweisilbige takt dem versanfange liegt. Nun bietet sich ja die sprache für eine gestalt öfter an als für eine andere (Tr in BB XVII 186), doch soll die folgende darlegung und dann die betrachtung des gesamt- $6 \mathrm{~T}$ zeigen, dals der dichter zum mindesten im allgemeinen gegen den versschlufs hin einsilbige takte $\mathrm{zu}$ gewinnen suchte. Einen zweisilbigen takt beliebiger form, $\therefore$ so gut wie $\mathcal{\sim} \sim$, konnte er nur am versanfang gebrauchen. Bei dieser freiheit beginnt der vers sehr häufig mit einem zweisilbigen takt, und z.t. mag diese häufigkeit bewirkt haben, dâs auch die im versinnern allein zulässige form '́ dem anfang mit vorliebe zugeschoben wurde; der letzte takt des verses endlich darf ja nur dann zweisilbig sein, wenn er auch sprachlich $=\stackrel{\text { L }}{ }$ ist. Scheinbar spricht gegen unseren satz, daIs gestalt 14 ( $\left.{ }^{\prime} \dot{\prime}^{\prime} \prime \prime\right)$ doppelt so häufig begegnet als 15 ('는). Die verhältnismälsige seltenheit von 15 erklärt sich daher, dafs diese gestalt im $6 \mathrm{~T}$ nur mit ' $\backsim$, nicht mit $\lrcorner_{\llcorner}$, d. h. nur mit einem wort wie gode oder heriges beginnen kann. Weit häutiger bietet sich da im $6 \mathrm{~T}$ die sprache für 14 an; ein wort mit langer stammsilbe, auf die zwei leichte silben irgend welcher art folgen, beginnt das Hst: dahin gehören auch wortverbindungen wie beornas on eaxlum, die als freie $4 \mathrm{~T}$ gerade so gut gestalt 15 sein könnten ${ }^{1}$ )

1) Gegen meine behauptung, dafs beornas on eaxlum als Hst des $6 \mathrm{~T}$ $=14$ sein müsse, lä́st sich nicht einwenden, hierfür fehle der beweis, 
(abgesehen natürlich von dem fall, dafs beornas einziges stabwort der betreffenden halbzeile wäre). Von dieser notgedrungenen grölseren häufigkeit von 14 gegenüber 15 abgesehen trifft der genannte satz zu: 14 und 15 zusammen sind

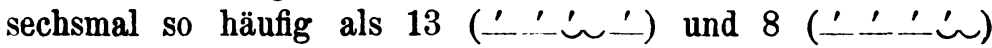
zusammen; 13 ist wieder weit häufiger als 8. Die gestalten mit vier und drei einsilbigen takten umfassen zusammen über neun zehntel aller Hst. Es verhält sich von ihnen hinsichtlich der häufigkeit $16: 14: 15:(13+8)$ etwa wie $6: 2: 1:{ }^{1} / 2$. Von den übrigbleibenden Hst entfällt nur ein teil auf die unter den gew. $V$ recht häufigen gestalten mit zwei einsilbigen takten; der rest fällt grofsenteils auf formen, die den "dehnversen" der freien $4 \mathrm{~T}$ gleichkommen, mit nur ein paar versen auf gestalten mit nur einem einsilbigen takte. Es bleiben schliefslich ein paar unsichere Hst. Die nur aus zweisilbigen takten bestehende gestalt 1 ist in den $6 \mathrm{~T}$ gar nicht belegt.

Wie stellt sich nun der $6 \mathrm{~T}$ als ganzes dar? Im allgemeinen ist die mehrzahl seiner takte einsilbig; die $6 \mathrm{~T}$ mit vier, fünf und sechs einsilbigen takten machen die grofse mehrheit aus; am häufigsten begegnen von diesen die mit vier einsilbigen takten; die mit fünf einsilbigen takten sind etwas weniger häufig, viel seltener sind die mit sechs einsilbigen takten. Von den versen mit drei oder weniger einsilbigen takten hat die überwiegende mehrzahl drei einsilbige takte. Die zweisilbigen takte finden sich vorzugsweise gegen den versanfang hin. So kommt es, dafs die grolse mehrzahl der Vst einen oder beide takte zweisilbig hat, während mehr als neun zehntel aller Hst ganz oder zu dreien von den vier takten aus einsilbigen takten bestehen. Die grundlage dieser verteilung ist die freiheit des versanfangs, mit einem zwei-

man wisse ja nicht, ob eine solche verbindung nicht auch im $6 \mathrm{~T}$ als 15 genommen worden sei. Der beweis liegt darin, dafs sich im $6 \mathrm{~T}$ kein Hst findet, das als $15 \mathrm{mit}$ dem ersten takt $=\stackrel{-}{ }$ genommen werden $\mathrm{mu} \mathbf{\mathrm { s }}$, sondern nur solche Hst, die als 14 genommen werden können: bei fünf silben, deren erste metrisch lang sein muls und von denen die vierte und fünfte je einen takt füllen müssen, ist im Hst des $6 \mathrm{~T}$ stets sowohl die $z$ weite wie die dritte leicht, d. h. kann metrisch kurz genommen werden, während im freien $4 \mathrm{~T}$ die zweite leicht sein, die dritte aber für sich allein durch ihre schwere einen ganzen takt beanspruchen kann, was notwendig 15 ergibt. 
silbigen takt beliebiger schwere zu beginnen, und die daraus entspringende neigung des verses, von seinem ende zweisilbige takte überhaupt möglichst fernzuhalten, um so mehr als der letzte takt des verses infolge eines für ihn besonders geltenden zwanges nur sehr selten zweisilbig sein kann. So zeigt uns der $6 \mathrm{~T}$ wie der gew. $\mathrm{V}$ das besondere rhythmische gesetz von der freiheit des verseingangs und der zunehmenden festigkeit gegen den versschlufs hin (für den gew. $\mathrm{V}$ vgl. hier $\mathrm{Tr}$ in $\mathrm{ABbl} \mathrm{V} 92 \mathrm{f}$. unter $\mathrm{L}$ ).

Hinsichtlich der stäbe bin ich der meinung Tr's (BB XVII 187), dafs sie nicht die hauptsache am verse sind, sondern "teils ein blofser schmuck, teils ein mittel, das der belebung des sinnes dient." Der schmuck wurde aber so angebracht, daIs er den vers "beherrschte", "übertönte" (Luick in PBB XV).

Im einzelen gilt für die stäbe im $6 \mathrm{~T}$ folgendes. Der anvers hat fast stets zwei stäbe. Diese fallen in der regel auf den ersten und dritten takt des verses; jedoch hat etwa ein neuntel der anverse die stäbe im dritten und fünften takt. Letztere stellung darf man bei ihrer verhältnismälsigen häufigkeit nicht mit Sievers einfach als "unregelmälsigkeit der alliteration" bezeichnen. Es war deshalb auch nicht nötig, sie oben in den anmerkungen jedesmal anzugeben. Diese stellung ist auffallend häufig im Guthlac A, wo etwa drei fünftel der anverse sie haben; sie fehlt gänzlich unter den denkmälern mit zahlreichen längeren versen nur im Traumgesicht. Die fälle, in denen die stäbe sich anders als auf eine der beiden angegebcnen arten verteilen, sind verhältnismäIsig selten. Trotz der vielen möglichkeiten umfassen sie nicht mehr als ein zehntel aller anverse, wobei einzele denkmäler sich durch ungewöhnliche stellungen überhaupt (ExeterDenksprüche) oder durch bevorzugung einer bestimmten ungewöhnlichen stellung (Daniel hat häufig die stäbe im zweiten und dritten takte des verses) auszeichnen. Als ausnahmen haben auch die fälle zu gelten, in denen der anvers nur einen stab (etwa 1 dutzend fälle, meist im dritten takt des verses) oder drei stäbe (nur ein paar sichere fälle) aufweist. - Der abvers hat einen stab. Dieser steht fast stets im dritten takte des verses. Selten, in etwa 35 fällen, trifft er den 
ersten takt. Überdies gehört von diesen fällen die mehrzahl, etwa zwei drittel, den Denksprüchen an, wo diese stellung didaktischen charakter hat, vgl. die allgemeinen bemerkungen zu den Denksprüchen; sie ist dort in den anmerkungen nicht verzeichnet. Die fälle aufserhalb der Denksprüche sind als ausuahmen zu betrachten. Ausnahmen sind auch die wenigen (etwa $1 / 2 \mathrm{dtz}$.) fälle, in denen der hauptstab einen anderen als den ersten oder dritten takt trifft; hier liefse sich überdies die stabstellung z.t. durch umstellung bessern. - Gekreuzte stabung kommt in ein paar fällen vor.

Einiges ist noch allgemein über die stäbe und ihre stellung zu bemerken. Während der viertaktige anvers nach belieben einen oder zwei stäbe haben kann, hat der sechstaktige anvers deren regelmälsig zwei. Wir sehen hier Luicks gesetz beobachtet, dals der vers von den stäben übertönt ${ }^{1}$ ) sein mufs: im $4 \mathrm{~T}$ genügt dazu ein stab, im $6 \mathrm{~T}$ sind bei dessen grölserer länge stets zwei stäbe dazu erforderlich. Und hier ist Luick zu berichtigen, denn auch wenn der sechstaktige anvers seinen ersten stab nicht gleich am anfang, sondern erst im dritten takte hat, folgt noch ein zweiter. Allerdings steht ja auch dann der erste takt noch etwas gegen den anfang hin, aber es mufs doch die blofse länge des verses den zweiten stab nötig gemacht haben, denn sonst gäbe es doch wohl etwas mehr sechstaktige anverse mit nur einem stab in der mitte. Allerdings mag auf die durchführung

1) $\mathrm{Tr}$ weist darauf hin, dafs die stäbe ihre bedeutung teilweise auch als "gedächtnisstützen" für den vortragenden scop gehabt haben werden. In der tat waren die verse durch die stäbe leichter im gedächtnis znsammenzuhalten als wenn sie dieses schmuckes entbehrt hätten, wie ähnlich in neueren gedichten der endreim das gedächtnis entlastet. Für die verteilung der stäbe und insbesondere für ihre vermehrung im $6 \mathrm{~T}$ gegenüber dem gew. $\mathrm{V}$ wird indes ihre benutzung als gedächtnisstützen im wesentlichen von gleichem einflufs gewesen sein wie die von ihnen nach Luick gewünschte eigenschaft des "übertönens": die erfüllung beider bedingungen durch das gleiche mittel mag man sich als ein "zusammengehaltensein", "umklammertsein" der langzeile durch die stäbe vorstellen. So ergeben sich aus der gleichen ursache, der anwendung der stäbe, verschiedene wirkungen: ein reiz für den zuhörenden und eine hilfe für den vortragenden, die beide nur durch eine in bestimmter weise geordnete, ein gewisses mindestmals der häufigkeit einhaltende verwendung dieses versschmuckes zu erreichen waren. 
zweier stäbe in jeder stellung die notwendig zwei stäbe fordernde gewöhnliche stellung eben als die gewöhnliche etwas eingewirkt haben. Dals nun der sechstaktige abvers nur einen stab aufweist, wird nach dem eben gesagten nicht genügend dadurch erklärt, dafs hier die gewöhnliche stellung des stabes im dritten takt der versmitte näher ist als die gewöhnliche stellung des ersten stabes im anverse. Vielmehr ist anzunehmen, dafs der abvers von den beiden stäben des anverses z.t. noch mitbeherrscht wird, dafs zur übertönung des abverses also der hauptstab eine gewisse verstärkung von aufsen erfährt. Aufserdem mag der gebrauch im gewöhnlichen viertaktigen abverse, der auch stets nur einen stab hat, der einführung eines zweiten stabes im sechstaktigen abvers einen gewissen widerstand entgegengesetzt haben. Schliefslich wird man $\mathrm{zu}$ bedenken haben, dais es dem dichter wohl zu schwer gewesen wäre, für jede zwölftaktige langzeile vier stabwörter zu finden; zwar entspräche der zwölftaktigen langzeile im vergleich zur achttaktigen, die im durchschnitt vielleicht $21 / 2$ stäbe hat, etwa die stabzahl 4, wenn man die verhältnisse der zeilenlängen und der stabzahlen einander gleichsetzen dürfte; doch versteht sich, dals die möglichkeit der anbringung von stäben in einer zeile nicht in demselben mafse zunimmt wie die taktzahl, sondern in geringerem. - Auch die beobachtung des gesetzes von der beherrschung durch die stäbe im $6 \mathrm{~T}$ weist auf die selbständige entwicklung dieser versart hin. Wäre einfach dem Hst ein Vst "vorgesetzt", so sollte man doch erwarten, dals das Hst für sich allein etwa die stabstellung des gew. $V$ aufwiese. - Nicht erklärlich macht uns das gesetz der übertönung, warum gerade nur eine stellung der stäbe im $6 \mathrm{~T}$ so auffallend bevorzugt wird, denn bei einer verschiebung der stäbe um einen takt nach hinten wäre doch die übertönung ziemlich genau so gut möglich: warum also nicht häufig stellung der stäbe im zweiten und vierten takte den an- und im vierten takte des abverses? Hierfür ist mir die erklärung, dals der dichter die möglichkeit einer mechanischen abtrennung eines $4 \mathrm{~T}$ fühlte und bewufst oder unbewufst diese mögliche zergliederung des $6 \mathrm{~T}$ durch die stabstellung zum ausdruck brachte: daher vor allem im an- wie im abvers ein stab am anfang des abtrennbaren $4 \mathrm{~T}$; es versteht sich, dals dann der erste stab 
des anverses nicht dem zweiten, sondern dem ersten takt des Vst zufiel.

Ziemlich lange hatten wir uns mit den fragen nach dem metrischen charakter der längeren verse $\mathrm{zu}$ beschäftigen, doch erst mit der beantwortung solcher fragen kann ein fester boden für die betrachtung aller alten rhythmischen denkmäler gewonnen werden: ist doch die grundbedingung für jede beschäftigung mit ihnen die möglichkeit, sie ihrem rhythmus entsprechend zu lesen. Von selbst stellt sich nun die weitere frage: wozu stehen denn mitten unter den durch ihre häufigkeit den namen "gewöhnlicher verse" verdienenden metrischen gebilden diese selteneren, manchmal ganz vereinzelten ${ }^{1}$ ), meist aber in kleineren oder grölseren gruppen ${ }^{2}$ ) auftretenden $6 \mathrm{~T}$ ? Wir haben gesehen, dals sie sich nicht etwa blofs durch grölsere silbenzahl, also gewissermalsen blofs graduell, sondern durch gröfsere taktzahl, d.i. wesentlich von den gew. $V$ unterscheiden. Also mul's sich der dichter bei gestaltung jedes längeren verses

1) Hier sei eine z. t. auch bei den folgenden ausführungen benutzbare übersicht über die vereinzelten $6 \mathrm{~T}$ gegeben. Die bedeutung der fragezeichen ergibt sich beim einsehen der betreffenden stellen des besonderen teils. a) Sachlich erklärbare verse: Christ und Satan 605 a (?); "Crist III" 1050 a, 1378 b (?), 1561 a (?); Daniel 59 a (?), 106 a (?); Elene 701 a (?), 1102 b (?), 1157 b (?), 1159 b (?), 1276 b; Exodus 411 b (?); 4. Gebet 61 a ; Der Menschen Gemüt $82 a$; Guthlac B 1131 b; Hymnus 40 a. b) Sachlich nicht erklürbare verse: Ardreas 216a, 1114a; Christ und Satan 605a (?); "Crist III" 1108a, 1305 b, 1360a, 1561 a (?); Elene 631 b, 701 a (?); Genesis A 899 a (?), 1172 a (?), 1198 a (?); Guthlac B 1198a (?); Salomon und Saturn $300 \mathrm{a}$ (?), 398b; Seefahrer $23 \mathrm{a}$; Wanderer $65 \mathrm{a}$.

^) Manche gruppen werden vollständiger oder entstehen durch z u erwartende $6 \mathrm{~T}$, die aber in der überlieferung vermifst werden [doch braucht nicht jede durch $4 \mathrm{~T}$ unterbrochene gruppe verderbt sein, vgl. anmerkung zu Daniel 206-07]. Auch diese zu erwartenden verse seien hier gegeben, wobei einzele zu $6 \mathrm{~T}$ ergänzte und als solche im besonderen teil aufgeführte verse hier fortfallen: "Crist III" 1426 a (?), 1427 a (?); Elene 668a (?); Genesis A 2856 b; Guthlac B 1131 a (?); Judith 1 (?), 62 b; Salomon und Saturn 451 b, 454, 457; Wunder der Schöpfung 101 (?); Vaterunser 1a, 3. - In dieser wie in den obigen übersichten sind die Denksprüche nicht berücksichtigt, über deren die überreste von gröfseren zusammenhängen darstellende einzelverse das im besonderen teil unter "Allgemeines zu den Denksprüchen", zweiter absatz, bemerkte zu vergleichen ist. 
des metrischen unterschiedes wohl bewu[st gewesen sein. $\mathrm{Er}$ fühlte also, dafs er mit der versart wechselte. Es sei gleich bemerkt, daIs damit nicht immer gesagt ist: er beabsichtigte, mit der versart zu wechseln, bevor er dies wirklich tat. Betrachtet man nun aber die $6 \mathrm{~T}$ in den einzelen gedichten unter vergegenwärtigung des gesamten gedankeninhaltes des gedichtes und besonders dieser verse, so erhält man vielfach den eindruck, dals der dichter absichtlich die längere versart benutzt hat, um das in ihr gesagte als ihm wichtig scheinend hervorzuheben. Diesem eindruck verfällt jeder, der ein altenglisches gedicht liest, in dem $6 \mathrm{~T}$ etwas reichlich auftreten. So gibt denn auch die wissenschaftliche literatur, die sich mit der altenglischen dichtung beschäftigt, vielfach diesen eindruck wieder. Häufig blieb es aber beim blofsen eindruck, und dieser hat zu einer verallgemeinerung geführt, die zwar meist das richtige treffen kann, aber zu falschem leiten mufs, wenn man zu kritischen zwecken von ihr ausgeht. Diese verallgemeinerung sei mit den worten von Sievers (Pauls Grundrifs II 875) gegeben: "Unter schwellversen versteht man eine speziell dem Westgermanischen eigene art längerer verse, welche vorwiegend gruppenweise bei feierlicher oder erregter rede zusammenstehen." In diesem urteil ist der allgemeine eindruck wiedergegeben, dafs die längeren verse um eines sachlichen zweckes willen gebraucht werden und eben deshalb vereinzelt nicht wohl verwendet werden können. Von diesem im allgemeinen allerdings richtigen satze durfte man sich aber bei der sammlung der $6 \mathrm{~T}$ nicht leiten lassen, vielmehr durfte hier stets nur die verslehre die frage entscheiden: $4 \mathrm{~T}$ oder $6 \mathrm{~T}$ ? Ein bestreben, wenn möglich nur sachlicb erklärbare gruppen der längeren verse zu erhalten, wäre unberechtigt. Verwerflich also ist es auch, wenn Sievers über verse, deren zugehörigkeit zu einer versart seine verslehre nicht entscheiden kann, den "charakter der in rede stehenden stelle eine andentung geben" lassen will. Da ich so manchen metrisch unanfechtbaren $6 \mathrm{~T}$ fand, der eine sachliche berechtigung sicher nicht hat, so durfte immer erst nach feststellung eines $6 \mathrm{~T}$ lediglich auf dem boden der verslehre nachträglich untersucht werden, ob mit seinem gebrauch ein sachlicher zweck verknüpft war. Und bei dieser untersuchung bin ich zu dem 
ergebnis gekommen, dals es $6 \mathrm{~T}$ gibt, die sachlich nicht erklärbar sind.

Befassen wir uns mit diesen versen zunächst. Ihre zahl ist kleiner als es auf den ersten blick scheint, denn, wie ich bei den einzelen denkmälern glaube nachgewiesen $\mathrm{zu}$ haben, finden sich auch vereinzelte verse mit sachlicher absicht in der selteneren form gegeben, allerdings z.t. an stellen, wo wir heute an eine hervorhebung kaum denken würden. Man mufs sich da etwas in den naiven sinn des sängers der vorzeit hineinfühlen. Wie aber sind nun sachlich nicht erklärbare $6 \mathrm{~T}$ zu deuten? Der dichter wufste, dafs es aufser dem gew. $V$ noch die andere kunstform des $6 \mathrm{~T}$ gab, dafs auch diese ihm zu gebote stand. Nun länft ihm gewissermafsen unter seinen händen ein vers unter, der sich nicht als $4 \mathrm{~T}$ entwickelt: satzform, rhythmus und stabreim stehen auch in seiner gedrungenen sprache nicht immer im gleichen verhältnis zueinander. Wenn ihm nun ein solcher vers unterläuft, so verschmäht er es nicht, ihn in die neben dem gew. $V$ mögliche form des $6 \mathrm{~T}$ zu giefsen, wenn für diesen die eben genannten einzelheiten in zusammenstimmung gebracht werden können. Als deutlichere beispiele solchen unterlaufens möchte ich anfïhren Der Menschen Geschicke 15 f., Salomon und Saturn $310 \mathrm{f}$. So wird sich noch mancher $6 \mathrm{~T}$, den ich als "zufällig" oder "untergeschlüpft" bezeichnet habe, erklären. Sachlich nicht erklärbare $6 \mathrm{~T}$ können sich aber auch im zusammenhang mit sachlich berechtigten finden. Es konnte einem dichter, wenn z. b. in einer langzeile der abvers sachlich eine hervorhebung verdiente, besser scheinen, auch den anvers in der längeren versart zu geben, als die langzeile zwischen die beiden versarten zu teilen ("Crist III" 889). Noch leichter kann es vorkommen, dafs nach einem erklärbaren sechstaktigen anvers auch der abvers und vielleicht noch folgende zeilen sechstaktig werden. Da mag der dichter einer art trägheitgesetz folgend die einmal eingeschlagene bahn des längeren verses eingehalten haben. Hierhin gehört Daniel 226-28, wohl auch Andreas 1820-21, und vielleicht auch die verse im Runenlied sind hier zu nennen (wo aber der erste $6 \mathrm{~T}$ nicht sachlich berechtigt, sondern durch die satzform untergelaufen wäre). So erklären sich die metrisch sicheren, aber nicht aus sachlichem grunde angewendeten $6 \mathrm{~T}$. 
Was nun die sachlich erklärbaren längeren verse betrifft, so sind ihrer viele, bei denen der sachliche zweck dem dichter zwar bewufst war, aber heute mehr oder minder schwer zn erkennen ist. So war ich bei manchen versen genötigt, nur vermutend ihren möglichen sachlichen zweck anzudeuten. Mögen die vermutungen auch teilweise kühn ausgefallen sein, es mufste doch bei jedem verse gefragt werden: konnte der dichter an dieser stelle mit der anwendung der längeren versart eine sachliche absicht verbinden? was kann diese absicht gewesen sein? Der versuch, hierauf $\mathrm{zu}$ antworten, wird doch für einige verse, deren zweck nicht sofort in die augen springt, diesen zweck ihrer anwendung gefunden haben.

In vielen denkmälern ist die anwenḍung der $6 \mathrm{~T}$ im ganzen ziemlich willkürlich. Weder der grundgedanke noch die einzelen höhepunkte werden sorgfältig zur hervorhebung herangezogen, vielmehr wird hier und da etwas dem dichter wichtiges in $6 \mathrm{~T}$ gegeben. Der geistlich gerichtete dichter verfährt hierbei manchmal recht äufserlich. Wo gott in seine darstellung tritt, da hebt er hervor: nennung gottes, preis gottes, bitte an gott, die göttliche strafe. Eher verstehen wir es, wenn er seinen gesang in der längeren versart mit einer feierlichen anrufung gottes beginnt (Vaterunser, 4. Gebet 1) oder mit einer ermahnung, lehre schlielst (Wanderer 111-115, Wunder der Schöpfung). Wo er eine "moral" bringt, wendet er überhaupt gern $6 \mathrm{~T}$ an (Seefahrer 106-09), sei es auch nur, um diese lehre in der längeren versart einzuleiten (Der Menschen Gemüt).

Vielfach aber ist die anwendung der $6 \mathrm{~T}$ eine sachlich gute, wo nämlich wirkliche höhepunkte der darstellung, sei es der handlung oder der betrachtung hervorgehoben werden. Diese höhepunkte lassen sich bei ihrem sehr verschiedenen charakter nicht weiter hier allgemein behandeln, doch war für sie bei betrachtung des zusammenhanges der zweck der hervorhebung leicht $\mathrm{zu}$ erkennen. Durch fast allseitig gate anwendung der $6 \mathrm{~T}$ zeichnen sich nur einige denkmäler aus. Von diesen stehen an der spitze das Traumgesicht und Judith; in beiden gedichten ist die ganze eigentliche handlung in $6 \mathrm{~T}$ gegeben. Eine etwas andere, aber ähnlich vortreffliche art der anwendung zeigt das gedicht von Guthlac dem Einsiedler, 
wo alle höhepunkte rhythmisch hervorgehoben sind. Ähnliches wie von diesem gedicht lälst sich von Daniel sagen, doch ist für ihn zu bemerken, dals - auch abgesehen von der "interpolation" 279-408 - nur in einem teile des gedichtes, in der geschichte der drei jünglinge im feurigen ofen, die höhepunkte so belebend gekennzeichnet sind, im übrigen gedicht aber stellen in $6 \mathrm{~T}$ fehlen. In allen übrigen denkmälern ist $\mathrm{zwar}$ auch $\mathrm{z}$. $\mathrm{t}$. wichtiges in den $6 \mathrm{~T}$ gegeben, nirgends aber findet man die längeren verse mit einem so allgemein wie in den eben genannten gedichten auf das wesentliche gerichteten blick angewendet. Durch vielfach berechtigte anwendung zeichnet sich noch "Crist III" aus. Die sicher von Cynewulf verfafsten gedichte zeigen ziemliche willkür im gebrauch der $6 \mathrm{~T}$, soweit sie deren überhaupt aufweisen. Es scheint, dals Cynewulf mit der längeren versart nicht viel anzufangen wufste.

Am schlufs dieser betrachtung über die anwendung unserer verse drängt sich noch die frage auf: gab es wohl auch gedichte, die ganz in der selteneren versform abgefafst waren? Eine bestimmte antwort auf diese frage läfst sich nicht geben, doch macht mich $\mathrm{zu}$ ihrer bejahung ein umstand geneigt, dessen bedeutung $\mathrm{zu}$ hoch $\mathrm{zu}$ veranschlagen ich allerdings nicht wage: die längeren verse in den Denksprüchen sind sicher $6 \mathrm{~T}$, und hinsichtlich ihres auftretens ist es von möglichen ausnahmen abgesehen gewils, dals sie nicht ihren inhalt gegen die umgebung hervorheben sollen, sondern selbsändige bedeutung haben und grolsenteils - in der ursprünglichen gestalt der sprüche vielleicht alle - zu sprüchen von wenigstens ursprünglich jedesmal einheitlicher sechstaktiger versform gehören.

Sechstakter und verfasserfragen. Zur entscheidung von verfasserfragen kann mittels unserer verse in dreifacher weise beigetragen werden; nämlich durch betrachtung 1 . ihrer häufigkeit, 2. der art ihrer anwendung, 3. der besonderheiten ihrer form. Die folgende liste gibt die in betracht kommenden denkmäler und bestimmt sie nach den drei gesichtspunkten, soweit diese bei dem einzelen denkmal $\mathrm{zu}$ verfasserfragen etwas aussagen können. "Gegen Cy." = unwahrscheinlichkeit von Cynewulfs verfasserschaft, die sich aus einem punkte er- 
gibt. Ergebnisse früherer forschung sind als solche durch angabe des namens in klammer angedeutet.

\begin{tabular}{|c|c|c|c|}
\hline & 1. Häufigkeit. & $\begin{array}{l}\text { 2. Artder } \\
\text { anwendung. }\end{array}$ & $\begin{array}{c}\text { 3. Besonderheit } \\
\text { der form. }\end{array}$ \\
\hline Cynewulf. Elene. & $11 / 5 \%$ & & \\
\hline Andreas. & $2 / 3 \%$ & $\begin{array}{l}\text { Ziemlich willkïr- } \\
\text { lich. }\end{array}$ & $\begin{array}{l}\text { Form ziemlich } \\
\text { durchschnittliche. }\end{array}$ \\
\hline Himmelfahrt. & $1 / 4 \%$ & & \\
\hline Juliane. & - . & & \\
\hline Guthlac B. & $11 / 2 \%$ & $\begin{array}{l}\text { Vielleicht etwas } \\
\text { besser als bei Cy. } \\
\text { Nicht so gut wie } \\
\text { in Guthl. A. }\end{array}$ & \\
\hline Guthlac A. & $\begin{array}{l}3^{3} / \mathrm{\%} \% \text { [vielleicht: } \\
\text { vf.] gegen Cy. (Tr) } \\
\text { und auch gegen ge- } \\
\text { meinsamkeit des ver- } \\
\text { fassers mit Guthl. B. }\end{array}$ & $\begin{array}{l}\text { Gut: gegen Cy. } \\
\text { und gegen gemein- } \\
\text { samkeit des verfas- } \\
\text { sers mit Guthl. B. }\end{array}$ & $\begin{array}{l}\text { Drei fünftel der } \\
\text { verse haben die st } \\
\text { in dritten und fünt } \\
\text { takt: gegen Cy. ( } \\
\text { und gegen gemeins } \\
\text { keit des verfassers } \\
\text { Guthl. B. }\end{array}$ \\
\hline "Crist III". & $\begin{array}{r}3^{1} / 2 \% \text { : [vielleicht: } \\
\text { vf.] gegen Cy. (Tr). }\end{array}$ & Besser als bei $\mathrm{Cy}$. & \\
\hline "Crist I". & $\begin{array}{l}\text { - : vielleicht ge- } \\
\text { gen gemeinsamkeit } \\
\text { des verfassers mit } \\
\text { "Crist III". }\end{array}$ & & \\
\hline Judith. & $\begin{array}{l}19 \% \text { : gegen } \mathrm{Cy} . \\
\text { (Tr). }\end{array}$ & Sehr gut: gegen Cy. & \\
\hline Traumgesicht. & $\begin{array}{l}21 \%: \text { gegen } \mathrm{Cy} . \\
(\mathrm{Tr})\end{array}$ & Sehr gut: gegen Cy. & \\
\hline Denkspruche. & $\begin{array}{l}\text { Exon. } 33^{\circ}{ }_{10}, \text { Cot- } \\
\text { ton. } 12^{\circ},{ }^{\circ} \text { : gegen } \\
\text { Cy. }\end{array}$ & $\begin{array}{l}\text { Ursprünglich ein- } \\
\text { zele sprüche einheit- } \\
\text { lich in } 6 \mathrm{~T} \text {, was } \mathrm{Cy} . \text { } \\
\text { der mit den } 6 \mathrm{~T} \\
\text { nicht viel anzufan- } \\
\text { gen weils, kaum zu- } \\
\text { zutrauen ist. }\end{array}$ & $\begin{array}{l}\text { Besonders in Es } \\
\text { auffallend ungereg } \\
\text { stabstellung, eini } \\
\text { verse von unregelmä } \\
\text { gem bau : tatsachen, } \\
\text { auf eine sicher } \\
\text { fremde volksmärsi } \\
\text { versbehandlung hi } \\
\text { weisen. }\end{array}$ \\
\hline
\end{tabular}




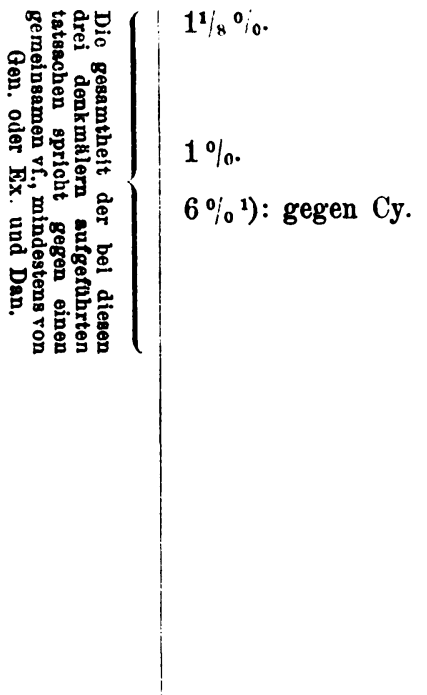

Willkiürlich.

Ziemlich gut.

Gut: gegen Cy.
Hst der gestalt 16 in drei vierteln der $6 \mathrm{~T}$ : vielleicht gegen $\mathrm{Cy}$.

Form die gewöhnliche.

Hst der gestalt 16 in knapp der hälfte der 6 T. - Ein achtel der anverse hat den ersten stab im zweiten takt: vielleicht gegen Cy.; für diese stabstellung auch in allen übrigen denkmälern zusammen nur noch ein paar beispiele. Ein fünftel der anverse hat die stäbe im dritten und fünften takt.

Die aufstellung bringt in verfasserfragen keine neue entscheidung, sie bestätigt nur bereits festgestelltes. Die beweiskraft der tatsachen unter 2 . und 3 . ist für sich allein nur bei starkem hervortreten dieser tatsachen von bedeutung; deshalb ist die von Sievers (PBB XII 465) vorgenommene zusammenfassung von Guthlac A, "Crist III" und Andreas wegen der in ihnen vorkommenden selteneren stellung der stäbe im dritten und fünften takte des anverses unstatthaft; diese stabstellung ist nur bei Guthlac A - und zwar gegen Cynewulfs verfasserschaft - beweisend. Unter 1. aber spricht gegen Cynewulf als vf. doch vielleicht schon ein plus von einigen prozent gegenüber Cynewulfs $6 \mathrm{~T}$-reichstem werk, der Elene, angesichts der tatsache, dals bei ihm, der doch in der verwendung der längeren verse nicht eben wählerisch ist, kein denkmal zu mehr als etwas über ein prozent aus solchen besteht: Cynewulf liebt die verse nur wenig. - G. Grau, Quellen

1) Steiner nimmt p. $51 \mathrm{f}$. das fehlen von "schwellversen" in Dan. 279408 als beweis für die interpolierung dieser stelle. Indes zeigt auch sonst der Daniel auf weite strecken keine längeren verse, und wenn auch die verse 279-408 gerade zwischen $6 \mathrm{~T}$-reiche strecken des übrigen gedichtes sich anschliel'sen, so ist doch zu bedenken, dafs an diesen $6 \mathrm{~T}$-reichen stellen der höhepunkt der geschichte von den drei jünglingen im feurigen ofen gegeben wird, während die verse 279-408 grörstenteils keine handlung, sondern gebete enthalten. Nur die stelle 33:3-361 entspricht hier jenem mit recht in $6 \mathrm{~T}$ gehaltenen höhepunkt. 
und Verwandtschaften der älteren germanischen Darstellungen des jüngsten Gerichtes (= heft XXXI der von Morsbach herausgegebenen Studien zur englischen Philologie), Halle 1908, kann hier nur mehr berührt werden. Auf grund seiner quellenforschungen weist Grau dem Cynewulf aufser den nach Tr sicher echten werken u. a. "Crist III" zu. Dafs in diesem die zahl der längeren verse grölser ist als selbst in der Elene, spricht nach G. (p. 43) nicht gegen gemeinsamen ursprung beider gedichte: die "schwellverse" seien in "Crist III" nicht gleichmäLsig verteilt, sie ständen am dichtesten, wo zu ihrer anwendung am meisten anlals gewesen sei, "in der aufs höchste erregten verdammungsrede an die zur linken"; wenn nun Elene hinsichtlich der häufigkeit der längeren verse zwischen Crist III und Crist I, II stehe, "so erklärt sich das vollauf aus der gleich [wie in "Crist III"] zornigen situation der hauptstelle in El. 582 - 589". Dazu ist zu nehmen, was Grau p. 92 zu Guthlac bemerkt: "Wer dem gebrauche der schwellverse gewicht beilegt, möge bedenken, dals in den lebhaft bewegten kämpfen der verse $1-7903,9 \%$, in 791-1353 1,7\% ... vorkommen, was zu Crist III (und El.) gut stimmt." Bei der vergleichung von "Crist III" und Elene hat G. nicht unrecht; doch bleibt mein eindruck, dafs "Crist III" in der sachlichen anwendung der längeren verse im allgemeinen über Cynewulf steht. Was aber Guthlac A betrifft, so mülste Cynewulf bei ähnlich guter anwendung der $6 \mathrm{~T}$ sicher einen höheren prozentsatz von diesen erreichen als dies selbst in der Elene der fall ist (hierbei ganz abgesehen von Cynewulfs willkürlichen $6 \mathrm{~T}$, deren wir in Guthlac A keine finden). - -

Zur bekräftigung der tatsache, dals die beiden gedichte von Salomon und Saturn weiter nichts als das auftreten derselben personen gemein haben, mag auch der umstand dienen, dafs nur das zweite gedicht $6 \mathrm{~T}$ hat: $6 \%$.

Ergänzungen und verbesserungen: p. 12 z. 17 v. u. hinter 1a lies punkt statt komma. - p. 32 z. 8 v. o. mülste 2856 b fett gedruckt sein. - p. 59 ist zu 40b die anmerkung zu ergänzen: Hauptstab im ersten takt des Vst. - p. 182 z. 19 v. u. lies blofs statt blos. - p. 189 z. 12 v. o. lies Heuslers statt Heulers.

Bons.

Theodor Schmitz. 